\title{
On the Existence of Rotating Stars in General Relativity
}

\author{
Uwe Heilig \\ Institut für Theoretische Physik der Universität Tübingen, Auf der Morgenstelle 14, \\ D-72076 Tübingen, Germany. e-mail: ptih101@compserv.zdv.uni-tuebingen.de
}

Received: 18 June 1993

Abstract: The Newtonian equations of motion, and Newton's law of gravitation can be obtained by a limit $\lambda=\frac{1}{c^{2}} \rightarrow 0$ of Einstein's equations. For a sufficiently small constant $\Lambda$ the existence of a set of solutions $(0 \leq \lambda \leq \Lambda)$ of Einstein's equations of a stationary, axisymmetric star is proven. This existence is proven in weighted Sobolev spaces with the implicit function theorem. Since the value of the causality constant $\lambda$ depends only on the units used to measure the velocity, the existence of a solution for any small $\lambda$ is physically interesting.

\section{Introduction}

In order to study the properties of a relativistic equilibrium stellar model, we would like to take the energy-momentum tensor of a bounded ideal fluid body and find the most general solution of Einstein's equations. Even in Newtonian fluid mechanics the ellipsoidal figures with constant density are essentially the only rotating solutions that are known [5]. In general relativity there exists no analytical solution representing a rotating star. Although there are known some interior solutions, they could not be extended to the exterior of the star. Thus, the question arises, under which circumstances there exists a solution of Einstein's equations representing a rotating star. One kind of existence theorem is given in this paper (Theorem 7.1).

In general relativity the spacetime of an isolated and stationary star has a timelike Killing vector $\partial_{t}$ that represents the symmetry relative to translations of time, and a spacelike Killing vector $\partial_{\varphi}$ that represents the symmetry relative to the axis of rotation. The star consists of a rigidly rotating ideal fluid, whose density and pressure are related by an equation of state. I would like to point to the fact that this stellar model follows from much more general conditions under the condition "thermodynamic equilibrium" [13].

In this paper the existence of solutions of Einstein's equations is proven, that fulfill the above restrictions. Precisely, we use Einstein's equations of Ehler's frame theory 
$[7,8]$ instead of Einstein's equations of general relativity. These equations contain a parameter $\lambda$, the causality constant. If $\lambda=0$, then these equations are equivalent to the Newtonian equations of motion and Newton's law of gravitation. If $\lambda=\frac{1}{c^{2}}$, then Einstein's equations of the frame theory are equivalent to Einstein's equations of general relativity. In Sect. 2 Einstein's equations of the frame theory are introduced. We assume that a solution of the equations in Newtonian fluid mechanics is given, i.e. we assume a given solution of Einstein's equations with causality constant $\lambda=0$. This solution represents a Newtonian star, rigidly rotating with angular velocity $\omega_{0}$, and consisting of ideal fluid. We prove the existence of constants $\Lambda, \Omega$, such that for all causality constants $0 \leq \lambda \leq \Lambda$, and all angular velocities $\left|\omega-\omega_{0}\right| \leq \Omega$ there are solutions of Einstein's equations of the frame theory. Since any solution of Einstein's equations of the frame theory with causality constant $\lambda \neq 0, \lambda \neq \frac{1}{c^{2}}$ can be considered a solution of Einstein's equations of general relativity, displayed in a system of units, in which the gravitational constant has the value $G$, and the speed of light has the value $\frac{1}{\sqrt{\lambda}}$, these solutions are of physical interest, even if $\Lambda<\frac{1}{c^{2}}$. This interpretation is discussed at the end of Sect. 2. I would like to point to the fact that there are known various equations of state, such that there exist solutions, representing a static $\left(\omega_{0}=0\right)$ Newtonian star. Thus, this existence theorem yields the existence of slowly rotating stars in general relativity.

To prove this existence theorem, some parts of mathematical theory are reviewed in Sect. 3. These are the Sobolev spaces, which guarantee some mathematical theorems (Sect. 3.1), the implicit function theorem, which is used to prove the existence of solutions in the neighbourhood of a given one (Sect. 3.2), and the properties of the components of Einstein's equations (Sect. 3.3).

Einstein's equations in the form used in this paper can be devided into 3 parts:

1. The equations of motion,

2. The "reduced field equations",

3. The harmonic conditions.

In Sect. 4 the reduced field equations are solved, if density and pressure of the fluid are given functions. In the Newtonian case $(\lambda=0)$, this can be done by pure integration (Sect. 4.1), and for $\lambda>0$, the implicit function theorem yields the existence of such solutions (Sect. 4.2). If the full Einstein equations for a stationary and axisymmetric energy-momentum tensor are solved, it holds that the metric also is stationary and axisymmetric, i.e. has the Killing vectors $\partial_{t}$, and $\partial_{\varphi}$. At the end of Sect. 4.2 it is shown that if pressure and density are stationary, and axisymmetric functions, the solution of the reduced field equations also represents a stationary, and axisymmetric spacetime. This has the consequence that the equations of motion are equivalent to the single Euler equation.

In Sect. 5 the existence of solutions of Euler's equation and of the reduced field equations in the neighbourhood of the Newtonian solution, introduced in Sect. 5.1, is shown with the implicit function theorem. To use the implicit function theorem, the differentiability of a function is needed. This causes some limitations for the equation of state (Sect. 5.2).

In Sect. 6 it is shown that for sufficiently small causality constants the harmonic conditions are fulfilled automatically, if the reduced field equations and the equations of motion are fulfilled. Thus, the solutions of the reduced field equations and 
Euler's equation are solutions of Einstein's equations. The result is summarized in Theorem 7.1.

\section{Einstein's Equations}

Let $\left(h^{\imath \jmath}\right)$ be a metric of a spacetime, and

$$
\left(g^{i j}\right):=\frac{1}{\sqrt{\left|\operatorname{det}\left(h^{i j}\right)\right|}}\left(h^{i \jmath}\right)
$$

the corresponding tensor density. In the case of a flat spacetime, there exists a coordinate system $(t, x, y, z)=\left(x^{0}, x^{1}, x^{2}, x^{3}\right)$ such that the metric has the form $\left(h_{0}^{\imath \jmath}\right)=\operatorname{diag}\left(-\frac{1}{c^{2}}, 1,1,1\right)$. In this chart the tensory density has the form

$$
\left.\underset{0}{g}=\underset{0}{\left(g^{\imath \jmath}\right.}\right)=\left(\begin{array}{cccc}
-\sqrt{\lambda} & 0 & 0 & 0 \\
0 & 1 / \sqrt{\lambda} & 0 & 0 \\
0 & 0 & 1 / \sqrt{\lambda} & 0 \\
0 & 0 & 0 & 1 / \sqrt{\lambda}
\end{array}\right), \quad \lambda:=\frac{1}{c^{2}}
$$

In order to describe the influence of matter on spacetime, we define the functions

$$
U^{i j}:=\frac{1}{4 \sqrt{\lambda}^{3}}\left(g^{\imath j}-g_{0}^{\imath \jmath}\right) .
$$

According to Lottermoser [14], Einstein's equations can then be written in the form

$$
4 \pi G|d| T^{\imath j}=\bar{g}^{k l} U_{, k l}^{i j}+\bar{g}^{\imath j} U_{, k l}^{k l}-2 U_{, k l}^{k(i} \bar{g}^{j) l}+A^{\imath \jmath}+B^{i \jmath}+C^{i j},
$$

where

$$
\begin{aligned}
& \bar{g}^{i j}:=\sqrt{\lambda} g^{\imath j}=\bar{g}_{0}^{\imath \jmath}+4 \lambda^{2} U^{\imath \jmath}, \\
& \bar{g}_{\imath j}:=\sqrt{\lambda} g_{\imath j} \\
& d:=\lambda \operatorname{det}\left(g^{\imath j}\right) \text {, } \\
& A^{\imath \jmath}:=2\left[\frac{1}{2} \bar{g}_{k l} \bar{g}_{m n}-\bar{g}_{n k} \bar{g}_{m l}\right]\left[\bar{g}^{i a} \bar{g}^{j b}-\frac{1}{2} \bar{g}^{\imath \jmath} \bar{g}^{a b}\right] U_{, a}^{k l} U_{, b}^{m n}, \\
& B^{i \jmath}:=4 \lambda \bar{g}_{k l}\left[2 \bar{g}^{n(i} U^{\jmath) l}{ }_{, m} U^{k m}{ }_{, n}-\frac{1}{2} \bar{g}^{i j} U^{k m}{ }_{, n} U^{l n}{ }_{, m}-\bar{g}^{m n} U^{\imath k}{ }_{, m} U^{j l}{ }_{, n}\right] \text {, } \\
& C^{\imath j}:=4 \lambda^{2}\left[U_{, k}^{i j} U_{, l}^{k l}-U_{, l}^{i k} U_{, k}^{j l}\right] \text {, }
\end{aligned}
$$

and $T^{i j}$ is the energy-momentum tensor of matter. Any solution $\left(\lambda, U^{\imath \jmath}, T^{\imath j}\right)$ of these equations for arbitrary $\lambda \geq 0$ is a solution of Ehler's frame theory $[7,8]$ with causality constant $\lambda$.

If $\lambda \neq 0$, the equations of motion, $\nabla_{i} T^{i j}=0$, follow from Bianchi's identities. However, if $\lambda=0$, the frame theory shows that they have to be imposed as an additional axiom. If we choose harmonic coordinates,

$$
\nabla_{i} \nabla^{i} x^{j}=0, \quad \text { or } \quad U_{, i}^{\imath \jmath}=0
$$


we can divide Einstein's equations of Ehler's frame theory into

1. the equations of motions, $\nabla_{i} T^{\imath \jmath}=0$,

2. the harmonic conditions, $U^{i j}{ }_{, 2}=0$,

3. the "reduced field equations", $4 \pi G|d| T^{\imath j}=F^{i j}$, where

$$
\begin{aligned}
F^{\imath j}= & \bar{g}^{k l} U^{i \jmath}{ }_{, k l}+4 \lambda^{2}\left[U^{k l} U^{\imath j}{ }_{, k l}+U^{i j} U^{k l}{ }_{, k l}-2 U^{k(\imath)}{ }_{, k l} U^{\jmath) l}\right] \\
& +A^{i j}+B^{\imath \jmath}+C^{\imath \jmath} .
\end{aligned}
$$

Due to the harmonic conditions we could drop the terms $U^{i \jmath} U^{k l}{ }_{k l}-2 U^{k(\imath}{ }_{, k l} U^{j) l}$ within the reduced field equations. However, if the equations of motion and the reduced field equations are fulfilled for certain energy-momentum tensors and sufficiently small causality constants $\lambda$, the harmonic conditions are satisfied (see Sect. 6).

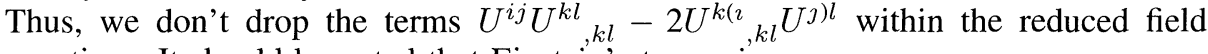
equations. It should be noted that Einstein's tensor is

$$
G^{i j}=\frac{2 \lambda^{2}}{|d|}\left(F^{i j}+\bar{g}_{0}^{\imath j} U_{, k l}^{k l}-2 U_{, k l}^{k(i} \bar{g}_{0}^{j) l}\right) .
$$

With

$$
\left(\bar{g}_{\imath \jmath}\right)=\left(\begin{array}{cccc}
-1 & 0 & 0 & 0 \\
0 & \lambda & 0 & 0 \\
0 & 0 & \lambda & 0 \\
0 & 0 & 0 & \lambda
\end{array}\right), \quad\left(\bar{g}^{\imath j}\right)=\left(\begin{array}{cccc}
-\lambda & 0 & 0 & 0 \\
0 & 1 & 0 & 0 \\
0 & 0 & 1 & 0 \\
0 & 0 & 0 & 1
\end{array}\right),
$$

and the formula

$$
\lim _{\lambda \rightarrow 0} \Gamma_{\jmath k}^{i}=\left\{\begin{array}{ll}
U^{00} & \text { for } j=k=0 \text { and } i \neq 0 \\
0 & \text { otherwise }
\end{array},\right.
$$

which can be derived with estimate (6.1), the limit $\lambda \rightarrow 0$ in Einstein's equations yields the Newtonian equations for the Newtonian potentials $\stackrel{N}{U^{\imath \jmath}}$ and the Newtonian energy-momentum tensor $\stackrel{N}{T}$.

$$
\text { 1. } \stackrel{N^{\prime}}{{ }^{i}{ }_{, 2}}= \begin{cases}0 & \text { for } j=0 \\ -U^{00}{ }_{, j} T^{00} & \text { for } j \neq 0\end{cases}
$$

2. $\stackrel{N}{U}^{\imath j}{ }_{, i}=0$

3. $\Delta \stackrel{N}{U^{i j}}=4 \pi G \stackrel{N}{T}^{i j}+\left(\left[\bar{g}^{i a}\right]_{\lambda=0}\left[\bar{g}^{\jmath b}\right]_{\lambda=0}-\frac{1}{2}\left[\bar{g}^{i j}\right]_{\lambda=0}\left[\bar{g}^{a b}\right]_{\lambda=0}\right) \stackrel{N}{U^{00}}{ }_{, a}^{N^{00}}{ }_{, b}$

$$
=4 \pi G T^{\imath \jmath}+ \begin{cases}U^{N} U^{00}{ }_{,}^{N} U^{00}{ }_{, j}-\frac{1}{2} \delta^{\imath \jmath}\left\|\nabla U^{N 0}\right\|^{2} & \text { for } i \neq 0 \text { and } j \neq 0 \\ 0 & \text { otherwise }\end{cases}
$$

According to [14, Sect. 3.3], Eqs. 1 and 3 are equivalent to the Newtonian equations of motion, and Newton's law of gravitation. If we use function spaces, such that the equations $f=0$ and $\Delta f=0$ are equivalent, the harmonic coniditions 2 follow from the equations of motion and the reduced field equations. Thus, Eqs. 1, 2, 3 can be regarded as Newtonian equations. 
In this paper we investigate the existence of a set of solutions of Einstein's equations (2.3) for $0 \leq \lambda \leq \Lambda$. We use the energy-momentum tensor of a star, consisting of ideal fluid, and rotating with angular velocity $\omega$. The density and the pressure of the matter are related by an equation of state. The spacetime of this stellar model is axisymmetric and stationary, i.e., there exists a timelike and a spacelike Killing vector, $\partial_{t}$ and $\partial_{\varphi}$, respectively.

The energy-momentum tensor of an ideal fluid, moving with velocity $v=\partial_{t}+$ $\omega \partial_{\varphi}$, is

$$
T^{i \jmath}=(\varrho+\lambda p) u^{2} v^{i} v^{\jmath}+p \frac{\bar{g}^{\imath j}}{\sqrt{|d|}}
$$

where

$$
\lambda u^{2} v_{\imath} v^{\imath}=-1, \quad u^{-2}=-\lambda h_{\imath \jmath} v^{\imath} v^{\jmath}=-\sqrt{|d|} \bar{g}_{\imath j} v^{i} v^{j} .
$$

In our harmonic coordinate system we use the following ansatz for the Killing vectors:

$$
\partial_{t}=(1,0,0,0), \quad \partial_{\varphi}=\left(0,-x^{2}, x^{1}, 0\right) .
$$

Since the coordinate lines of $x^{0}$ are integral curves of the Killing vector $\partial_{t}$, all functions are independent of $x^{0}$.

Because $v$ is a Killing vector, and $v^{\imath} \nabla_{i} \varrho=v^{i} \nabla_{i} p=0$, the equations of motion $\nabla_{\imath} T^{\imath j}=0$ are equivalent to Euler's equation

$$
0=p_{, \imath}+(\varrho+\lambda p) \frac{1}{2 \lambda} \partial_{x^{\imath}}\left(\ln \left(u^{-2}\right)\right) .
$$

At the end of this section we will discuss a possible physical meaning of a solution of Einstein's equations (2.3) for $0 \neq \lambda \neq \frac{1}{c^{2}}$. Any solution of Eqs. (2.3) can be considered a solution of Einstein's equations of general relativity, displayed in a new system of units in which the gravitational constant has the value $G$ and the speed of light has the value $\frac{1}{\sqrt{\lambda}}$. We will denote all quantities in the new system with a prime. The transformation

$$
\begin{aligned}
& \text { new system } \rightarrow \text { SI-system } \\
& \text { length: } \quad l^{\prime} \rightarrow l:=\frac{\alpha}{c \sqrt{\lambda}} l^{\prime} \\
& \text { time: } \quad t^{\prime} \rightarrow t:=\alpha t^{\prime} \\
& \text { mass: } \quad m^{\prime} \rightarrow m:=\frac{\alpha}{c^{3} \sqrt{\lambda}^{3}} m^{\prime}
\end{aligned}
$$

with any $\alpha>0$ transforms this new system of units into the SI-system. Transformation of pressure $\left([p]=\frac{k g}{m s^{2}}\right)$, density $\left([\varrho]=\frac{k g}{m^{3}}\right)$, and angular velocity $\left([\omega]=\frac{1}{s}\right)$, yields

$$
\varrho^{\prime}=\frac{1}{\alpha^{2}} \varrho, \quad p^{\prime}=\frac{1}{c^{2} \lambda \alpha^{2}} p, \quad \text { and } \quad \omega^{\prime}=\frac{1}{\alpha} \omega,
$$

respectively. Thus, this transformation changes the form of the equation of state to $p(\varrho)=\alpha^{2} c^{2} \lambda p^{\prime}\left(\frac{\varrho}{\alpha^{2}}\right)$. 
If we are interested in solving Einstein's equations of general relativity for a given equation of state $p(\varrho)$, we must take a set of equations of state $p_{\lambda}^{\prime}\left(\varrho^{\prime}\right)$, such that we can solve Einstein's equations of frame theory for any $\lambda \neq 0$ with equation of state $p_{\lambda}^{\prime}\left(\varrho^{\prime}\right)$. Then, we can consider this solution a solution of Einstein's equations of general relativity with equation of state $p(\varrho)=\alpha^{2} c^{2} \lambda p_{\lambda}^{\prime}\left(\frac{\varrho}{\alpha^{3}}\right)$, displayed in the new system of units. Thus, we must take such a set $p_{\lambda}^{\prime}$, that for an appropriate choice of $\alpha$, the desired equation of state can be achieved.

A physical interesting equation of state with $p(0)=0$ can be written in the form

$$
p(\varrho)=\varrho^{\nu} f(\varrho),
$$

where $f(0) \neq 0$ and $\nu>1$. If we take the set of equations of state

$$
p_{\lambda}^{\prime}\left(\varrho^{\prime}\right)=\varrho^{\prime \nu} f\left(\left(c^{2} \lambda\right)^{\frac{1}{\nu-1}} \varrho^{\prime}\right),
$$

then $\lim _{\lambda \rightarrow 0} p_{\lambda}^{\prime}\left(\varrho^{\prime}\right)=f(0) \varrho^{\prime \nu}$. It follows that the set of equations of state depends regularly on the parameter $\lambda$, and we can expect the limitations to be fulfilled, we have to impose on the equation of state (Sect. 5.2). Thus, with the existence Theorem 7.1, we get a set of solutions of Eqs. (2.3) for an energy-momentum tensor of a rotating body, consisting of ideal fluid with $p_{\lambda}^{\prime}\left(\varrho^{\prime}\right)$. Since we consider a solution with $\lambda \neq 0$ a solution of Einstein's equations, displayed in the new system of units, this solution has the following equation of state in the SI-system:

$$
p(\varrho)=\alpha^{2} c^{2} \lambda\left(\frac{\varrho}{\alpha^{2}}\right)^{\nu} f\left(\left(c^{2} \lambda\right)^{\frac{1}{\nu-1}} \frac{\varrho}{\alpha^{2}}\right) .
$$

If we choose $\alpha=\left(c^{2} \lambda\right)^{\frac{1}{2(\nu-1)}}$, this solution is a solution of Einstein's equations of general relativitiy with equation of state

$$
p(\varrho)=\varrho^{\nu} f(\varrho)
$$

and angular velocity

$$
\omega=\left(c^{2} \lambda\right)^{\frac{1}{2(\nu-1)}} \omega^{\prime}
$$

displayed in the new system of units.

\section{Weighted Sobolev Spaces and Functional Analysis}

\subsection{Weighted Sobolev Spaces}

Definition 3.1. Let $\|\cdot\|_{p}$ be the standard norm of $L^{p}\left(\mathbb{R}^{n}\right)$, and $\sigma(x):=\sqrt{|x|^{2}+1}$. Then, the Banach space $M_{m, \delta}^{p}\left(\mathbb{R}^{n}\right)$ is the completion of $C_{0}^{\infty}\left(\mathbb{R}^{n}\right)$ with respect to the norm

$$
\|f\|_{m, p, \delta}:=\sum_{|\alpha| \leq m}\left\|\sigma^{\delta+|\alpha|} \partial^{\alpha} f\right\|_{p} .
$$

Here $\alpha$ is a multi-index, and $C_{0}^{\infty}\left(\mathbb{R}^{n}\right)$ is the set of $C^{\infty}$-functions with compact support.

This definition is due to Cantor [2]. In this paper, Cantor investigated the bijectivity of the Laplacian on weighted Sobolev spaces. An improvement in the analysis of 
the Laplacian was achieved by McOwen [15]. Theorem 1 of his paper and the corresponding proof yields

Proposition 3.2. For all $n \geq 3$ and $-\frac{n}{p}<\delta<-2+n\left(1-\frac{1}{p}\right)$ the Laplacian

$$
\Delta: M_{2, \delta}^{p}\left(\mathbb{R}^{n}\right) \rightarrow M_{0, \delta+2}^{p}\left(\mathbb{R}^{n}\right)
$$

is bijective and continuous. The inverse operator $\Delta^{-1}$ is

$$
\left[\Delta^{-1} f\right](x)=\frac{1}{(2-n) A_{n}} \int_{\mathbb{R}^{n}} \frac{f\left(x^{\prime}\right)}{\left|x-x^{\prime}\right|^{n-2}} d x^{\prime n},
$$

where $A_{n}$ is the area of the unit sphere in $\mathbb{R}^{n}$.

According to Cantor [3, Theorem 5.6], the following proposition holds:

Proposition 3.3. For all $\delta \leq \delta_{1}+\delta_{2}, m \leq m_{1}, m_{2}$ with $m<m_{1}+m_{2}-\frac{n}{p}$ there exists a constant $C$, such that for all $f \in M_{m_{1}, \delta_{1}}^{p}\left(\mathbb{R}^{n}\right)$ and all $g \in M_{m_{2}, \delta_{2}}^{p}\left(\mathbb{R}^{n}\right)$ it holds that

$$
\|f g\|_{m, p . \delta} \leq C\|f\|_{m_{1}, p, \delta_{1}}\|g\|_{m_{2}, p, \delta_{2}} .
$$

Thus, the pointwise multiplication

$$
\begin{array}{ccccc}
M_{m_{1}, \delta_{1}}^{p}\left(\mathbb{R}^{n}\right) & \times & M_{m_{1}, \delta_{2}}^{p}\left(\mathbb{R}^{n}\right) & \rightarrow & M_{m, \delta}^{p}\left(\mathbb{R}^{n}\right), \\
f & \times & g & \rightarrow & f \cdot g
\end{array}
$$

is bilinear and continuous.

\subsection{Differentiability and Analyticity}

Definition 3.4. $\mathscr{L}_{2}\left(B_{1}, B_{2}\right)$ denotes the Banach space of $i$-linear and continuous maps from the Banach space $B_{1}$ into the Banach space $B_{2}$ with the norm

$$
\|f\|_{\mathscr{L}_{i}\left(B_{1}, B_{2}\right)}=\sup \left\{\left\|f\left(x_{1}, \ldots, x_{\imath}\right)\right\|_{B_{2}} ; \sup \left\{\left\|x_{1}\right\|_{B_{1}}, \ldots,\left\|x_{i}\right\|_{B_{1}}\right\} \leq\right\} .
$$

Definition 3.5. $C^{k}\left(A, B_{2}\right)$ is the set of functions, which map the open subset $A$ of the Banach space $B_{1}$ into the Banach space $B_{2}$, and are $k$ times Fréchet differentiable. The derivative of $f$ at a point $x_{0}$ is denoted with $D f\left(x_{0}\right)$. If $f$ has several arguments, $D_{x^{3}} f\left(x_{1}, \ldots, x_{i}\right)$ denotes the partial derivative with respect to the argument $x^{j}$.

A function $f: A \rightarrow B_{2}$ is said to be of class $C^{\omega}\left(A, B_{2}\right)$, if for each $x_{0} \in A$ there is a radius $r>0$ and a sequence $f_{i} \in \mathscr{L}_{i}\left(B_{1}, B_{2}\right)$ of $i$-linear symmetric maps, such that

$$
\sum_{i=0}^{\infty}\left\|f_{i}\right\|_{\mathscr{E}_{\imath}\left(B_{1}, B_{2}\right)} r^{i}<\infty
$$

and for all $x$ with $\left\|x-x_{0}\right\|<r$ it holds that

$$
f(x)=\sum_{\imath=0}^{\infty} f_{\imath} \underbrace{\left(x-x_{0}, \ldots, x-x_{0}\right)}_{i \text { times }} \text {. }
$$




\section{Remarks.}

- A derivative $D f\left(x_{0}\right)$ of a function $f$ at a point $x_{0}$ belongs to $\mathscr{L}\left(B_{1}, B_{2}\right)$.

- Higher derivatives at $x_{0}$ into directions $h_{1}, \ldots, h_{k}$ are denoted with $D^{k} f\left(x_{0}, h_{1}, \ldots, h_{k}\right)$.

- If $B_{1}=\mathbb{R}^{n}, B_{2}=\mathbb{R}$, we have

$$
f_{i}\left(x-x_{0}, \ldots, x-x_{0}\right)=\sum_{|\alpha|=i} \frac{1}{\alpha !} \partial^{\alpha} f\left(x_{0}\right)\left(x-x_{0}\right)^{\alpha},
$$

and Definition 3.5 reduces to the statement that a function is called analytic if it can be represented by a uniformly and absolutely convergent power series.

- A function $f$ is of class $C^{\omega}\left(B_{R}(0), B_{2}\right)$, if for all $x \in B_{R}(0)$,

$$
f(x)=\sum_{i=0}^{\infty} f_{\imath}(x, \ldots, x) \text { with } \sum_{i=0}^{\infty}\left\|f_{i}\right\|_{\mathscr{L}_{i}\left(B_{1}, B_{2}\right)} R^{\imath}<\infty,
$$

where all $f_{i}$ are $i$-linear and symmetric. The proof is similar to that of the corresponding statement in the Banach space $\mathbb{R}$, since for all $x_{0} \in B_{R}(0)$ and all $h \in B_{R-\left\|x_{0}\right\|}(0)$ it holds that

$$
\begin{aligned}
f\left(x_{0}+h\right) & =\sum_{i=0}^{\infty} f_{i}\left(x_{0}+h, \ldots, x_{0}+h\right) \\
& =\sum_{i=0}^{\infty} \sum_{k=0}^{i}\left(\begin{array}{l}
i \\
k
\end{array}\right) f_{\imath}(\underbrace{x_{0}, \ldots, x_{0}}_{k \text { times }}, \underbrace{h, \ldots, h}_{i-k \text { times }}), \\
\sum_{\imath=0}^{\infty} & \sum_{k=0}^{\imath}\left(\begin{array}{l}
i \\
k
\end{array}\right)\left\|f_{i}\left(x_{0}, \ldots, x_{0}, h, \ldots, h\right)\right\|_{B_{2}} \\
& \leq \sum_{i=0}^{\infty} \sum_{k=0}^{i}\left(\begin{array}{l}
i \\
k
\end{array}\right)\left\|f_{i}\right\|_{\mathscr{S}_{i}\left(B_{1}, B_{2}\right)}\left\|x_{0}\right\|_{B_{1}}^{k}\|h\|_{B_{1}}^{2-k} \\
& \leq \sum_{i=0}^{\infty}\left\|f_{i}\right\|_{\mathscr{C}_{\imath}\left(B_{1}, B_{2}\right)}\left(\left\|x_{0}\right\|_{B_{1}}+\|h\|_{B_{1}}\right)^{\imath}<\infty .
\end{aligned}
$$

Linear and $i$-linear continuous maps are of class $C^{\omega}$. The derivative of $i$-linear functions into direction $h$ at $\left(x_{1}, \ldots, x_{i}\right)$ can be calculated by the product rule

$$
D f\left(x_{1}, \ldots, x_{i}\right) h=f\left(h_{1}, x_{2}, \ldots, x_{i}\right)+\ldots+f\left(x_{1}, \ldots, x_{i-1}, h_{\imath}\right) .
$$

- Concatenation of $C^{k}$, and $C^{\omega}$-functions yields a $C^{k}$-, and a $C^{\omega}$-function, respectively. The derivative can be calculated by the chain rule

$$
D(f \circ g)\left(x_{0}\right)=D f\left(g\left(x_{0}\right)\right) \cdot D g\left(x_{0}\right) .
$$

- $C^{\omega}\left(A, B_{2}\right) \subset C^{\infty}\left(A, B_{2}\right)$.

Proposition 3.6. Let $f: \mathbb{R}^{n} \rightarrow \mathbb{R}$ with $f(0)=0$ be analytic on the ball $B_{r}(0)$. Furthermore, let $B$ be a Banach space with a scalar multiplication and a commutative multiplication $B \times B \rightarrow B$, such that for a constant $C$ and all $X_{1}, X_{2} \in B$,

$$
\left\|X_{1} \cdot X_{2}\right\|_{B} \leq C\left\|X_{1}\right\|_{B} \cdot\left\|X_{2}\right\|_{B} \text {. }
$$


Then, the function

$f^{\prime}: B^{n} \rightarrow B, \quad\left(X_{1}, \ldots, X_{n}\right) \rightarrow f^{\prime}\left(X_{1}, \ldots, X_{n}\right):=\sum_{\alpha \geq 1}^{\infty} \frac{1}{\alpha !} \partial^{\alpha} f(0) X_{1}^{\alpha_{1}} \ldots X_{n}^{\alpha_{n}}$ is of class $C^{\omega}\left(B_{R}(0) \times \ldots \times B_{R}(0), B\right)$, where $R=\frac{r}{C}$.

Proof. With respect to the estimates

$$
\begin{gathered}
\left\|X_{1}^{\alpha_{1}} \ldots X_{n}^{\alpha_{n}}\right\|_{B} \leq C^{|\alpha|-1}\left\|X_{1}\right\|_{B}^{\alpha_{1}} \ldots\left\|X_{n}\right\|_{B}^{\alpha_{n}} \leq C^{|\alpha|-1} R^{|\alpha|} \\
\left\|\sum_{\alpha \geq 1}^{\infty} \frac{1}{\alpha !} \partial^{\alpha} f(0) X_{1}^{\alpha_{1}} \ldots X_{n}^{\alpha_{n}}\right\|_{B} \leq \frac{1}{C} \sum_{\alpha \geq 1}^{\infty} \frac{1}{\alpha !}\left|\partial^{\alpha} f(0)\right| r^{|\alpha|}
\end{gathered}
$$

this proposition easily follows from the fact, that for all $x \in B_{r}(0)$ we can represent $f$ by the absolutely convergent power series

$$
f(x)=\sum_{\alpha \geq 1}^{\infty} \frac{1}{\alpha !} \partial^{\alpha} f(0) x^{\alpha} .
$$

Major tools, to be used in the remainder of the paper, are the following theorems $[6,16]$ :

Theorem 3.7 (Taylor). Let $A$ be an open, convex domain of the Banach space $B_{1}$. If the function $f$ is in $C^{k+1}\left(A, B_{2}\right)$, then for any $x_{0}, h \in A$ it holds that

$$
\begin{aligned}
& f\left(x_{0}+h\right)=f\left(x_{0}\right)+\sum_{i=1}^{k} \frac{1}{i !} D^{i} f\left(x_{0}, h, \ldots, h\right)+R_{k+1}, \\
& R_{k+1}=\int_{0}^{1} \frac{(1-t)^{k}}{k !} D^{k+1} f\left(x_{0}+t h, h, \ldots, h\right) d t, \\
&\left\|R_{k+1}\right\|_{B_{2}} \leq \frac{1}{(k+1) !} \sup _{0 \leq t \leq 1}\left\|D^{k+1} f\left(x_{0}+t h, h, \ldots, h\right)\right\|_{B_{2}} .
\end{aligned}
$$

Theorem 3.8 (Implicit Function Theorem). Let $B_{1}, B_{2}, B_{3}$ be Banach spaces, and $A$ an open subset of $B_{1} \times B_{2}$. Furthermore, let

$$
f: A \rightarrow B_{3}, \quad(x, y) \rightarrow f(x, y)
$$

be a continuous function, whose partial derivative $D_{y} f(x, y)$ is continuous in $A$. Assume that for a point $\left(x_{0}, y_{0}\right) \in A$ it holds that $f\left(x_{0}, y_{0}\right)=0$ and $D_{y} f\left(x_{0}, y_{0}\right)$ is bijective. Then, there exists an open neighbourhood $L \subset B_{1}$ of $x_{0}$, and a continuous map $l: L \rightarrow B_{2}$, such that for all $x \in L$,

$$
f(x, l(x))=0 .
$$

In addition, it holds that $l \in C^{k}\left(L, B_{2}\right)$ if $f \in C^{k}\left(A, B_{3}\right)$. 


\subsection{Properties of the Tensor Density $\left(U^{i j}\right)$}

\subsubsection{The Function Space of the Tensor Density $\left(U^{\imath \jmath}\right)$}

Definition 3.9. For $p \geq 4$ and $0 \leq \delta<-2+3 \frac{p-1}{p}$

$$
\begin{aligned}
& F_{2}:=M_{2, \delta}^{p}\left(\mathbb{R}^{3}\right), \\
& F_{1}:=M_{1, \delta+1}^{p}\left(\mathbb{R}^{3}\right), \\
& F_{0}:=M_{0, \delta+2}^{p}\left(\mathbb{R}^{3}\right),
\end{aligned}
$$

are the Banach spaces of 2, 1, 0 times weakly differentable functions, respectively.

For a matrix $\left(U^{\imath \jmath}\right)$ of functions,

$$
\begin{aligned}
& F_{2}^{*}:=\left\{\left(U^{\imath j}\right) ; U^{\imath j} \in F_{2} \text { for all } i, j=0,1,2,3\right\}, \\
& F_{1}^{*}:=\left\{\left(U^{i j}\right) ; U^{i j} \in F_{1} \text { for all } i, j=0,1,2,3\right\}, \\
& F_{0}^{*}:=\left\{\left(U^{i j}\right) ; U^{i j} \in F_{0} \text { for all } i, j=0,1,2,3\right\}
\end{aligned}
$$

are the corresponding Banach spaces of $4 \times 4$ matrices with the norms

$$
\begin{aligned}
\left\|\left(U^{\imath \jmath}\right)\right\|_{F_{2}^{*}} & :=\sup _{\imath, \jmath=0, \ldots, 3}\left\|U^{\imath j}\right\|_{F_{2}}, \\
\left\|\left(U^{\imath \jmath}\right)\right\|_{F_{1}^{*}} & :=\sup _{\imath, j=0, \ldots, 3}\left\|U^{i j}\right\|_{F_{1}}, \\
\left\|\left(U^{\imath \jmath}\right)\right\|_{F_{0}^{*}} & :=\sup _{i, \jmath=0, \ldots, 3}\left\|U^{i j}\right\|_{F_{0}},
\end{aligned}
$$

respectively.

We can easily see that any smooth matter density $\delta$ with compact support is in $F_{0}$, the corresponding Newtonian potential $U$ is in $F_{2}$, and all components of the corresponding Newtonian gravitational force $-U_{, i}(i=1,2,3)$ are in $F_{1}$.

Properties of $F_{0}, F_{1}$, and $F_{2}$ :

1. Since all derivatives of $\sigma^{-1}$ are bounded, and $M_{m, \delta}^{p}\left(\mathbb{R}^{n}\right) \subset W^{m, p}\left(\mathbb{R}^{n}\right)$ for $\delta \geq 0$, we get with Sobolev's embedding theorem [1, Theorem 5.4], the following continuous embeddings for $0 \leq \nu<1-\frac{3}{p}$ :

$$
F_{2} \rightarrow C^{1, \nu}\left(\overline{\mathbb{R}}^{3}\right), \quad F_{1} \rightarrow C^{0, \nu}\left(\overline{\mathbb{R}}^{3}\right) .
$$

Here, $C^{i, \nu}$ denotes the Hölder space of $i$ times Hölder continuously differentiable functions.

2. With the Rellich-Kondrachov theorem [1, Theorem 6.2] we see, that for all compact domains $T \subset \mathbb{R}^{3}$ and all $0 \leq \nu<1-\frac{3}{p}$ the embeddings

$$
F_{2} \mapsto C^{1, \nu}(T), \quad F_{1} \mapsto C^{0, \nu}(T)
$$

are compact. It should be noted that an embedding $B_{1} \mapsto B_{2}$ is called compact, if it is continuous, and if all sequences that are bounded with respect to the norm $\|\cdot\|_{B_{1}}$, have a subsequence that converges with respect to $\|\cdot\|_{B_{2}}$. 
3. Due to Proposition 3.2, the operator

$$
\Delta: F_{2} \rightarrow F_{0}
$$

is linear and continuous. The inverse operator $\Delta^{-1}$ is

$$
\Delta^{-1}: F_{0} \rightarrow F_{2}, \quad f \rightarrow \frac{-1}{4 \pi} \int_{\mathbb{R}^{3}} \frac{f\left(x^{\prime}\right)}{\left|x-x^{\prime}\right|} d x^{\prime 3} .
$$

4. With Proposition 3.3 the following pointwise multiplications are bilinear and continuous:

$$
\begin{array}{ll}
F_{2} \cdot F_{2} \rightarrow F_{2}, & F_{1} \cdot F_{1} \rightarrow F_{1}, \\
F_{2} \cdot F_{1} \rightarrow F_{1}, & F_{1} \cdot F_{1} \rightarrow F_{0}, \\
F_{2} \cdot F_{0} \rightarrow F_{0}, & F_{1} \cdot F_{0} \rightarrow F_{0} .
\end{array}
$$

Furthermore, the multiplications

$$
\begin{aligned}
& F_{2}^{*} \cdot F_{2}^{*} \rightarrow F_{2}^{*}, \\
& F_{1}^{*} \cdot F_{1}^{*} \rightarrow F_{1}^{*},
\end{aligned}
$$

defined by the usual matrix multiplication, are bilinare and continuous.

5. Definition 3.1 of weighted Sobolev spaces yields the continuity of the differential operators

$$
\begin{aligned}
& \delta_{x^{2}}: F_{2} \rightarrow F_{1}, \\
& \delta_{x^{i}}: F_{1} \rightarrow F_{0} .
\end{aligned}
$$

\subsubsection{Properties of the Components of Einstein's equations}

In this section we investigate the differentiability of some components of Einstein's equations.

Proposition 3.10. Let $B_{\vartheta}(0)$ denote the ball with radius $\vartheta$, centered at the origin of $F_{2}^{*}$. Furthermore, let $B_{\varepsilon}(0)$ be the ball of radius $\varepsilon$, centered at the origin of $F_{2}$. Then, for any $\vartheta>0$ and any $\varepsilon>0$, there is $a \Lambda>0$, such that the following functions are of class $C^{\omega}$ :

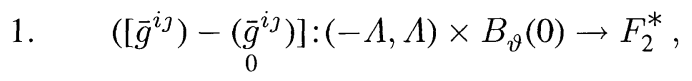
$\left(\lambda,\left(U^{i j}\right)\right) \rightarrow\left(\bar{g}^{i \jmath}\right)-\underset{0}{\left(\bar{g}^{\imath \jmath}\right),}$
2. $\left(\left[\bar{g}^{\imath j}\right)-\left(\bar{g}_{\imath j}\right)\right]:(-\Lambda, \Lambda) \times B_{\vartheta}(0) \rightarrow F_{2}^{*}$,
$\left(\lambda,\left(U^{i j}\right)\right) \rightarrow\left(\bar{g}_{\imath j}\right)-\left(\bar{g}_{0}\right)$,
3.

$$
[|d|-1]:(-\Lambda, \Lambda) \times B_{\vartheta}(0) \rightarrow B_{\varepsilon}(0),
$$$$
\left(\lambda,\left(U^{i j}\right)\right) \rightarrow|d|-1,
$$

The functions $\left(\bar{g}^{i j}\right),\left(\bar{g}_{i j}\right)$, and $d$ are defined in (2.2). If $\varepsilon$ is chosen sufficiently small, then the following functions are also of class $C^{\omega}$ :

1.

2.

3.

$$
\begin{array}{cl}
{[\sqrt{|d|}-1]:(-\Lambda, \Lambda) \times B_{\vartheta}(0) \rightarrow F_{2},} & \left(\lambda,\left(U^{\imath j}\right)\right) \rightarrow \sqrt{|d|}-1, \\
{\left[\frac{1}{\sqrt{|d|}}-1\right]:(-\Lambda, \Lambda) \times B_{\vartheta}(0) \rightarrow F_{2},} & \left(\lambda,\left(U^{i j}\right)\right) \rightarrow \frac{1}{\sqrt{|d|}}-1, \\
{\left[\frac{1}{|d|}-1\right]:(-\Lambda, \Lambda) \times B_{\vartheta}(0) \rightarrow F_{2},} & \left(\lambda,\left(U^{\imath \jmath}\right)\right) \rightarrow \frac{1}{|d|}-1 .
\end{array}
$$


Furthermore, the partial derivatives with respect to the argument $\left(U^{i \jmath}\right)$, evaluated at $\lambda=0$, vanish for all these functions.

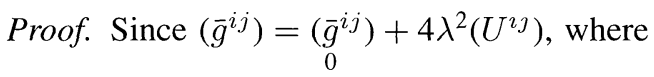

$$
\left(\bar{g}^{i j}\right)=\left(\begin{array}{cccc}
-\lambda & 0 & 0 & 0 \\
0 & 1 & 0 & 0 \\
0 & 0 & 1 & 0 \\
0 & 0 & 0 & 1
\end{array}\right)
$$

the first statement is trivial.

Let $U$ be an abbreviation for the matrix $\left(U^{i j}\right)$. With Born's formula $(\mathbb{1 1}-U)^{-1}=$ $\sum_{u=0}^{\infty} U^{\imath}$, it holds that

$$
\begin{aligned}
& \left.g^{-1}=\underset{0}{g}+4 \sqrt{\lambda^{3}} U\right)^{-1} \\
& \left.=\underset{0}{g}\left[\mathbb{1 1}+4 \sqrt{\lambda^{3}} g_{0}^{-1} U\right]\right)^{-1} \\
& =\left[11-\left(-4 \sqrt{\lambda^{3}} g_{0}^{-1} U\right)\right]_{0}^{-1} g_{0}^{-1} \\
& =\sum_{n=0}^{\infty}(-4 \lambda)^{n}\left(\sqrt{\lambda} g_{0}^{-1} U\right)^{n} g_{0}^{-1} \text {. }
\end{aligned}
$$

Thus

$$
\left(\bar{g}_{\imath j}\right)=\sqrt{\lambda} g^{-1}=\left[\sum_{n=0}^{\infty}(-4 \lambda)^{n}\left(\sqrt{\lambda} g^{-1} U\right)^{n}\right] \underset{0}{\sqrt{\lambda} g^{-1}}
$$

Since

$$
\left.\underset{0}{\sqrt{\lambda} g^{-1}}\right)=\underset{0}{\left(\bar{g}_{i \jmath}\right)}=\left(\begin{array}{cccc}
-1 & 0 & 0 & 0 \\
0 & \lambda & 0 & 0 \\
0 & 0 & \lambda & 0 \\
0 & 0 & 0 & \lambda
\end{array}\right)
$$

for any $A \in F_{2}^{*}$, and $|\lambda| \leq 1$, it holds that

$$
\left\|\sqrt{\lambda} g^{-1} A\right\|_{F_{2}^{*}} \leq\|A\|_{F_{2}^{*}}
$$

Because the multiplication $F_{2}^{*} \cdot F_{2}^{*} \rightarrow F_{2}^{*}$ is continuous, there exists a constant $C$, such that for any $A, B \in F_{2}^{*}$ it holds that

$$
\|A \cdot B\|_{F_{2}^{*}} \leq C\|A\|_{F_{2}^{*}} \cdot\|B\|_{F_{2}^{*}} .
$$

These inequalities yield

$$
\begin{aligned}
\| \underset{0}{\left(\sqrt{\lambda} g^{-1} 4 \lambda U\right)^{n} \|_{F_{2}^{*}}} & \leq C^{n-1}\left\|\sqrt{\lambda} g^{-1} 4 \lambda U\right\|_{F_{2}^{*}}^{n} \\
& \leq C^{n-1}(4 \lambda)^{n}\|U\|_{F_{2}^{*}}^{n} \\
& \leq C^{n-1}(4 \lambda \vartheta)^{n}
\end{aligned}
$$


Thus, the series (3.2) converges absolutely for $4 \lambda \vartheta C<1$, i.e., it converges for all $|\lambda| \leq \Lambda$ and all $\left(U^{\imath \jmath}\right) \in B_{\vartheta}(0)$ if $\Lambda<\frac{1}{4 \vartheta C}$. Hence,

$$
\left(\bar{g}_{\imath \jmath}\right)-\underset{0}{\left(\bar{g}_{\imath \jmath}\right)}=\left[\sum_{n=1}^{\infty}(-4 \lambda)^{n}\left(\sqrt{\lambda} g^{-1} U\right)^{n}\right] \underset{0}{\sqrt{\lambda} g^{-1}}
$$

is of class $C^{\omega}\left((-\Lambda, \Lambda) \times B_{\vartheta}(0), F_{2}^{*}\right)$, because it can be represented by an absolutely convergent power series.

According to [14, Eq. 3.15], it holds that

$$
\begin{aligned}
|d|= & -\lambda \operatorname{det}\left(g^{i j}\right) \\
= & 1-4 \lambda U+4 \lambda^{2} \operatorname{tr} Z-\lambda^{3} 16\left(U \operatorname{tr} Z-\vec{W}^{2}\right)+\lambda^{4} 8\left(\operatorname{tr}^{2} Z-\operatorname{tr} Z^{2}\right) \\
& -64 \lambda^{5}\left(U \frac{1}{2}\left(\operatorname{tr}^{2} Z-\operatorname{tr} Z^{2}\right)-\vec{W}^{2} \operatorname{tr} Z+\vec{W} Z \vec{W}\right) \\
& +64 \lambda^{6} \operatorname{det} Z-256 \lambda^{7}(U \operatorname{det} Z-\vec{W} \operatorname{adj}(Z) \vec{W}) .
\end{aligned}
$$

Here, we use Lottermoser's definitions

$$
\begin{aligned}
U & :=U^{00}, \\
\vec{W} & :=\left(U^{01}, U^{02}, U^{03}\right), \\
Z & :=\left(\begin{array}{lll}
U^{11} & U^{12} & U^{13} \\
U^{12} & U^{22} & U^{23} \\
U^{13} & U^{23} & U^{33}
\end{array}\right),
\end{aligned}
$$

and adj denotes the adjoint matrix. Thus, the continuity of the multiplication $F_{2} \cdot F_{2} \rightarrow$ $F_{2}$, and the fact that $|d|-1$ is a finite linear combination of products of the functions $U^{\imath j}$, lead to the existence of a $\Lambda>0$, such that the functions $[|d|-1]$ is of class $C^{\omega}\left((-\Lambda, \Lambda) \times B_{\vartheta}(0), B_{\varepsilon}(0)\right)$.

The functions $\sqrt{1+x}-1, \frac{1}{\sqrt{1+x}}-1$, and $\frac{1}{1+x}-1$ are analytic in the intervall $(-1,1)$, and vanish at $x=0$. Thus, Proposition 3.6 yields the existence of an $\varepsilon$, such that the functions

$$
\begin{gathered}
{[\sqrt{1+x}-1]: B_{\varepsilon}(0) \rightarrow F_{2}, \quad f(x) \rightarrow \sqrt{1+f(x)}-1,} \\
{\left[\frac{1}{\sqrt{1+x}}-1\right]: B_{\varepsilon}(0) \rightarrow F_{2}, \quad f(x) \rightarrow \frac{1}{\sqrt{1+f(x)}}-1,} \\
{\left[\frac{1}{1+x}-1\right]: B_{\varepsilon}(0) \rightarrow F_{2}, \quad f(x) \rightarrow \frac{1}{1+f(x)}-1}
\end{gathered}
$$

are of class $C^{\omega}\left(B_{\varepsilon}(0), F_{2}\right)$. Thus, since concatenation of $C^{\omega}$-functions yields a $C^{\omega}$ function, the functions

$$
[\sqrt{|d|}-1], \quad\left[\frac{1}{\sqrt{|d|}}-1\right], \quad \text { and } \quad\left[\frac{1}{|d|}-1\right],
$$

are of class $C^{\omega}\left((-\Lambda, \Lambda) \times B_{\vartheta}(0), F_{2}\right)$.

The statement that the partial derivatives with respect to the argument $\left(U^{i j}\right)$, evaluated at $\lambda=0$, vanish, easily follows with the power series representations of these functions and the chain rule. 
Since $v=\left(1,-\omega x^{2}, \omega x^{1}, 0\right)$, and the angular velocity $\omega$ is a constant, the components of the velocity $v$ are unbounded. However, the velocity $v$ is of interest only within the star. Thus, it is useful to choose a radius $R_{0}$, such that all matter of any body, regarded in this paper, lies within the ball $B_{R_{0}}(0)$. Then, we take a function $\xi \in C_{0}^{\infty}\left(\mathbb{R}^{3}\right)$, depending only on $|x|$, with $\xi(x)=1$ for $|x| \leq R_{0}$ and $\xi(x)=0$ for $|x| \geq 2 R_{0}$, and substitute $v$ by

$$
v:=\left(1,-\xi \omega x^{2}, \xi \omega x^{1}, 0\right)
$$

Using this definition of $v$, we have

Proposition 3.11. Let $B_{\vartheta}(0)$ denote the ball with radius $\vartheta$, centered at the origin of $F_{2}^{*}$. Furthermore, let $B_{\Theta}(0)$ be the ball of radius $\Theta$, centered at the origin of $F_{0}$. Then, for any $\vartheta>0$, any $\Theta>0$, and any $\Omega>0$, there is $a \Lambda>0$, such that the following functions are of class $C^{\omega}$ :

$$
\begin{aligned}
& \text { 1. }\left[u^{-2}-1\right]:(-\Lambda, \Lambda) \times(-\Omega, \Omega) \times B_{\vartheta}(0) \rightarrow F_{2}, \quad\left(\lambda, \omega,\left(U^{i j}\right)\right) \rightarrow\left[u^{-2}-1\right], \\
& \text { 2. }\left[u^{2}-1\right]:(-\Lambda, \Lambda) \times(-\Omega, \Omega) \times B_{\vartheta}(0) \rightarrow F_{2}, \quad\left(\lambda, \omega,\left(U^{\imath \jmath}\right)\right) \rightarrow\left[u^{2}-1\right] \text {, } \\
& \text { 3. }\left[\frac{1}{2 \lambda} \ln \left(u^{-2}\right)\right]:(-\Lambda, \Lambda) \times(-\Omega, \Omega) \times B_{\vartheta}(0) \rightarrow F_{2}, \quad\left(\lambda, \omega,\left(U^{i j}\right)\right) \rightarrow \frac{1}{2 \lambda} \ln \left(u^{-2}\right) \text {. }
\end{aligned}
$$

The function $u^{-2}$ is defined in (2.6). Additionally, the function

$$
\begin{aligned}
& {\left[\left(T^{i j}\right)\right]:\left((-\Lambda, \Lambda) \times(-\Omega, \Omega) \times B_{\vartheta}(0) \times B_{\Theta}(0) \times B_{\Theta}(0)\right) \rightarrow F_{0}^{*},} \\
& \quad\left(\lambda, \omega,\left(U^{\imath \jmath}\right), \varrho, p\right) \rightarrow\left(T^{i \jmath}\right),
\end{aligned}
$$

defined by the energy-momentum tensor

$$
T^{i j}=(\varrho+\lambda p) u^{2} v^{i} v^{j}+p \frac{\bar{g}^{\imath j}}{\sqrt{|d|}}, \quad \text { where } v=\left(1,-\xi \omega x^{2}, \xi \omega x^{1}, 0\right),
$$

is of class $C^{\omega}$.

Furthermore, the partial derivatives with respect to the argument $\left(U^{i j}\right)$, evaluated at $\lambda=0$, vanish for all these functions, except

$$
D_{\left(U^{i j}\right)}\left[\frac{1}{2 \lambda} \ln \left(u^{-2}\right)\left(0,\left(U^{i j}\right)\right)\right]\left(\delta U^{i \jmath}\right)=\delta U^{00} .
$$

Proof. With Eq. (3.4), we have

$$
\begin{aligned}
\bar{g}_{\imath \jmath} v^{i} v^{\jmath}= & \bar{g}_{00}-\bar{g}_{01} 2 \xi \omega x^{2}+2 \bar{g}_{02} \xi \omega x^{1}+\bar{g}_{11} \xi^{2} \omega^{2}\left(x^{2}\right)^{2} \\
& -2 \bar{g}_{12} \xi^{2} \omega^{2} x^{1} x^{2}+\bar{g}_{22} \xi^{2} \omega^{2}\left(x^{1}\right)^{2}
\end{aligned}
$$

The properties of $\xi$, the continuity of the multiplication $F_{2} \cdot F_{2} \rightarrow F_{2}$, Proposition 3.10 , and the equality $\bar{g}_{\imath \jmath}=\operatorname{diag}(-1, \lambda, \lambda, \lambda)$, imply that there exists a $\Lambda$, such that the
function

$$
\bar{g}_{i j} v^{i} v^{\jmath}+1
$$

is of class $C^{\omega}\left((-\Lambda, \Lambda) \times(-\Omega, \Omega) \times B_{\vartheta}(0), F_{2}\right)$. With Proposition 3.10 and the product rule it follows that

$$
\begin{aligned}
u^{-2}-1 & =-\sqrt{|d|} \bar{g}_{i j} v^{\imath} v^{\jmath}-1 \\
& =-(\sqrt{|d|}-1)\left(\bar{g}_{2 j} v^{i} v^{j}-1\right)-(\sqrt{|d|}-1)-\left(\bar{g}_{2 j} v^{\imath} v^{j}+1\right)
\end{aligned}
$$

is of class $C^{\omega}\left((-\Lambda, \Lambda) \times(-\Omega, \Omega) \times B_{\vartheta}(0), F_{2}\right)$. 
Proposition 3.10 and Eq. (3.5) show that the partial derivative of $u^{-2}-1$ with respect to the argument $\left(U^{i j}\right)$ vanishes at $\lambda=0$ for all $\left(U^{\imath \jmath}\right) \in B_{\vartheta}(0)$. Using Taylor's Theorem 3.7, for all $\left(U^{i \jmath}\right) \in B_{\vartheta}(0)$ it holds that

$$
\left[u^{-2}-1\right]\left(\lambda,\left(U^{\imath \jmath}\right)\right)=\left[u^{-2}-1\right)(\lambda, 0)+\int_{0}^{1} D_{\left(U^{i \jmath}\right)}\left[\left[u^{-2}-1\right]\left(\lambda, t\left(U^{i \jmath}\right)\right)\right]\left(U^{i \jmath}\right) d t .
$$

Because $\left[u^{-2}-1\right]$ and its derivative depend continuously on $\lambda$, it follows that for all $\varepsilon>0$, there exists a $\Lambda$, such that for all $|\lambda| \leq \Lambda$ and all $\left(U^{\imath j}\right) \in B_{\vartheta}(0)$ we have $\left\|u^{-2}-1\right\|_{F_{2}}<\varepsilon$. Thus, for all $\varepsilon>0$ there exists a $\Lambda$ such that $u^{-2}-1$ is of class $C^{\omega}\left((-\Lambda, \Lambda) \times(-\Omega, \Omega) \times B_{\vartheta}(0), B_{\varepsilon}(0)\right)$.

Since the functions $\frac{1}{1+x}-1, \ln (1+x)$ are analytic in $(-1,1)$ and vanish at $x=0$, Proposition 3.6 yields the existence of an $\varepsilon$, such that the functions

$$
\begin{gathered}
{\left[\frac{1}{1+x}-1\right]: B_{\varepsilon}(0) \subset F_{2} \rightarrow F_{2}, \quad f(x) \rightarrow \frac{1}{1+f(x)}-1,} \\
{[\ln (1+x)]: B_{\varepsilon}(0) \subset F_{2} \rightarrow F_{2}, \quad f(x) \rightarrow \ln (1+f(x))}
\end{gathered}
$$

are of class $C^{\omega}\left(B_{\varepsilon}(0), F_{2}\right)$. Since concatenation of $C^{\omega}$-functions yields a $C^{\omega}$-function,

$$
\left[u^{-2}-1\right], \quad \text { and }\left[\ln \left(u^{-2}\right)\right]
$$

are of class $C^{\omega}\left((-\Lambda, \Lambda) \times(-\Omega, \Omega) \times B_{\vartheta}(0), F_{2}\right)$ for sufficiently small $\Lambda$. A calculation of the power series of $\ln \left(u^{-2}\right)$ yields

$$
\left.\ln \left(u^{-2}\right)=-\lambda \xi^{2} \omega^{2}\left(\left(x^{1}\right)^{2}+\left(x^{2}\right)^{2}\right)\right)+2 \lambda U^{00}(x)+O\left(\lambda^{2}\right),
$$

where $O\left(\lambda^{2}\right)$ represents all elements of the power series, that are at least quadratic in $\lambda$. Since there exists a $\Lambda$, such that this power series converges absolutely for all $|\lambda| \leq \Lambda$, the power series that represents $\frac{1}{2 \lambda} \ln \left(u^{-2}\right)$,

$$
\left.\frac{1}{2 \lambda} \ln \left(u^{-2}\right)=-\xi^{2} \frac{\omega^{2}}{2}\left(\left(x^{1}\right)^{2}+\left(x^{2}\right)^{2}\right)\right)+U^{00}(x)+O(\lambda),
$$
also converges for these $\lambda$. Thus $\left[\frac{1}{2 \lambda} \ln \left(u^{-2}\right)\right]$ is of class $C^{\omega}((-\Lambda, \Lambda) \times(-\Omega, \Omega) \times$
$\left.B_{\vartheta}(0), F_{2}\right)$.

With

$$
\left.T^{\imath \jmath}=(\varrho+\lambda p)\left(u^{2}-1\right) v^{i} v^{j}+p\left(\frac{\bar{g}^{\imath j}}{\sqrt{|d|}}-\underset{0}{\bar{g}^{i \jmath}}\right)+(\varrho+\lambda p) v^{\imath} v^{j}+\underset{0}{\left(\bar{g}^{\imath j}\right.}\right)
$$

and the continuity of the multiplications $F_{2} \cdot F_{2} \rightarrow F_{2}, F_{2} \cdot F_{0} \rightarrow F_{0}$, the product rule leads to the analyticity of $T^{i j}$. Finally, the statements about the derivatives at $\lambda=0$ follow by straightforward calculations.

Remarks. I would like to point out that there are some regions, where the functions of Propositions 3.10, 3.11 are defined mathematically, but where they are physically absurd. One example is $\lambda<0$. Later, we restrict ourself to those regions, where $\lambda \geq 0$, and to those densities $\varrho$ and pressures $p$, where $T^{i j}$ has the meaning of an energy-momentum tensor of a bounded body, consisting of ideal fluid, and rotating uniformly with angular velocity $\omega$. 
Without proof, I present the results of the calculation of the first elements of the power series, representing the functions, used in Propositions 3.10, 3.11:

$$
\begin{aligned}
|d|-1 & =-4 \lambda U^{00}+O\left(\lambda^{2}\right) \in F_{2}, \\
\sqrt{|d|}-1 & =-2 \lambda U^{00}+O\left(\lambda^{2}\right) \in F_{2}, \\
\frac{1}{\sqrt{|d|}}-1 & =2 \lambda U^{00}+O\left(\lambda^{2}\right) \in F_{2}, \\
\left(\bar{g}_{\imath \jmath}\right)-\left(\bar{g}_{\imath \jmath}\right) & =-4 \lambda\left(\delta_{\imath}^{0} \delta_{\jmath}^{0}\right) U^{00}+O\left(\lambda^{2}\right) \in F_{2}^{*}, \\
u^{-2}-1 & =2 \lambda\left[-\xi^{2} \frac{\omega^{2}}{2}\left(\left(x^{1}\right)^{2}+\left(x^{2}\right)^{2}\right)+U^{00}\right]+O\left(\lambda^{2}\right) \in F_{2}, \\
u^{2}-1 & =2 \lambda\left[\xi^{2} \frac{\omega^{2}}{2}\left(\left(x^{1}\right)^{2}+\left(x^{2}\right)^{2}\right)-U^{00}\right]+O\left(\lambda^{2}\right) \in F_{2}, \\
\frac{1}{2 \lambda} \ln \left(u^{-2}\right) & =-\xi^{2} \frac{\omega^{2}}{2}\left(\left(x^{1}\right)^{2}+\left(x^{2}\right)^{2}\right)+U^{00}+O(\lambda) \in F_{2} .
\end{aligned}
$$

\section{Solution of the Reduced Field Equations}

\subsection{Solution of the Newtonian Field Equations}

For $\lambda=0$ and any $\left(U^{\imath \jmath}\right) \in F_{2}^{*}$ we have $\frac{1}{\sqrt{|d|}}-1=0$ and $u^{2}-1=0$. Thus, the Newtonian limit $\lambda \rightarrow 0$ in the energy-momentum tensor

$$
T^{\imath \jmath}=(\varrho+\lambda p) u^{2} v^{\imath} v^{\jmath}+p \frac{\bar{g}^{\imath \jmath}}{\sqrt{|d|}}
$$

yields the Newtonian energy-momentum tensor

$$
\stackrel{N}{T^{i j}}=\varrho v^{\imath} v^{\jmath}+\underset{0}{p\left[\bar{g}^{\imath \jmath}\right]_{\lambda=0}},
$$

where

$$
\left[\left(\bar{g}^{i j}\right)\right]_{\lambda=0}=\left(\begin{array}{cccc}
0 & 0 & 0 & 0 \\
0 & 1 & 0 & 0 \\
0 & 0 & 1 & 0 \\
0 & 0 & 0 & 1
\end{array}\right), \quad v=\left(1,-\xi \omega x^{2}, \xi \omega x^{1}, 0\right)
$$

Inserting this equation into the newtonian field equations (2.5) leads to

$$
\begin{aligned}
\Delta U^{i j}= & 4 \pi G\left(\varrho v^{\imath} v^{\jmath}+\underset{0}{p\left[\bar{g}^{\imath \jmath}\right]_{\lambda=0}}\right)+\stackrel{N}{U^{00, \imath}} \stackrel{N}{U}^{00, \jmath} \\
& -\frac{1}{2} \underset{0}{\left[\bar{g}^{i j}\right]_{\lambda=0}} \stackrel{N}{U}^{00, a}{ }^{N} U_{, a}^{N 0},
\end{aligned}
$$

when using the following definition: 
Definition 4.1. For any differentiable function,

$$
f^{, i}:=\underset{0}{\left[\bar{g}^{i j}\right]_{\lambda=0} f_{, \jmath}}= \begin{cases}\partial_{x^{i}} f & \text { for } i \neq 0 \\ 0 & \text { for } i=0\end{cases}
$$

denotes the partial derivative of $f$, raised with the matrix $\left[\left(\bar{g}_{0}^{i j}\right)\right]_{\lambda=0}$.

Since the Laplacian $\Delta: F_{2} \rightarrow F_{0}$ is continuous and has the inverse (3.1), these equations are equivalent to

$$
\begin{aligned}
& \stackrel{N}{U^{\imath \jmath}}(x)
\end{aligned}
$$

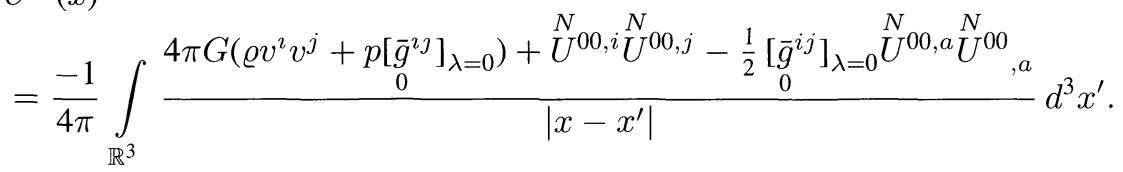

We can solve these equations by pure integration, because for $i=j=0$ it reads

$$
\stackrel{N}{U}^{00}(x)=-G \int_{\mathbb{R}^{3}} \frac{\varrho\left(x^{\prime}\right)}{\left|x-x^{\prime}\right|} d^{3} x
$$

Since this is the equation for the Newtonian potential of the density $\varrho$, we see that the Newtonian field equations and Newton's law of gravitation are equivalent.

With the continuity of the operations $\Delta^{-1}: F_{0} \rightarrow F_{2}, \partial_{x^{\imath}}: F_{2} \rightarrow F_{1}$, and the continuity of the multiplications $F_{1} \cdot F_{1} \rightarrow F_{0}, F_{2} \cdot F_{0} \rightarrow F_{0}$, we can convince ourselves that for all $\Theta>0$ and all $\Omega>0$ there exists a constant $C$, such that for any $\varrho, p \in B_{\Theta}(0) \subset F_{0}$, and any $\omega \in[-\Omega, \Omega]$, the solution of the Newtonian field equations fulfills

$$
\left\|\left(\stackrel{N}{U}^{\imath \jmath}\right)\right\|_{F_{2}^{*}} \leq C
$$

\subsection{The Case $\lambda \neq 0$}

Because of (3.1), the reduced field equations (2.3) are equivalent to

$$
U^{i j}(x)=\frac{-1}{4 \pi} \int_{\mathbb{R}^{3}} \frac{4 \pi G\left|d\left(x^{\prime}\right)\right| T^{i j}\left(x^{\prime}\right)-\left[F^{i j}\left(x^{\prime}\right)-\Delta U^{i j}\left(x^{\prime}\right)\right]}{\left|x-x^{\prime}\right|} d^{3} x^{\prime} .
$$

Proposition 4.2 (Solution of the Field Equations). Let $B_{\Theta}(0)$ and $B_{\vartheta}(0)$ denote the balls, centered at the origin of $F_{0}$ with radius $\Theta$ and $F_{2}^{*}$ with radius $\vartheta$, respectively. For all $\Theta>0, \Omega>0$, there exist constants $\Lambda>0, \vartheta>0$, and a differentiable function

$$
\left[\left(U^{i j}\right)\right]:(-\Lambda, \Lambda) \times(-\Omega, \Omega) \times B_{\Theta}(0) \times B_{\Theta}(0) \rightarrow B_{\vartheta}(0) \subset F_{2}^{*},
$$

such that $\left[\left(U^{i j}\right)\right](\lambda, \omega, \varrho, p)$ and the energy-momentum tensor

$$
T^{i j}=(\varrho+\lambda p) u^{2} v^{\imath} v^{\jmath}+p \frac{\bar{g}^{\imath \jmath}}{\sqrt{|d|}}
$$

solve Eqs. (4.4) and the reduced field equations. 
Proof. Due to estimate (4.3), there exists a constant $C$, such that for all $\varrho, p \in B_{\Theta}$, and all $\omega \in[-\Omega, \Omega]$, the solutions of the Newtonian field equations lie in $B_{C}(0) \subset F_{2}^{*}$. Choose $\vartheta=2 C$.

Proposition 3.10 , the continuity of the operators

$$
\begin{array}{ll}
\partial_{x^{i}}: F_{2} \rightarrow F_{1}, & F_{2} \cdot F_{0} \rightarrow F_{0}, \\
\partial_{x^{i}}: F_{1} \rightarrow F_{0}, & F_{1} \cdot F_{1} \rightarrow F_{0},
\end{array}
$$

and the product rule lead to the existence of a $\Lambda$, such that

- $\left[\left(A^{\imath \jmath}\right)\right]:(-\Lambda, \Lambda) \times B_{\vartheta}(0) \rightarrow F_{0}^{*}, \quad\left(\lambda,\left(U^{i \jmath}\right)\right) \rightarrow\left(A^{\imath \jmath}\right)$,

- $\left[\left(B^{\imath j}\right)\right]:(-\Lambda, \Lambda) \times B_{\vartheta}(0) \rightarrow F_{0}^{*}, \quad\left(\lambda,\left(U^{\imath j}\right)\right) \rightarrow\left(B^{i j}\right)$,

- $\left[\left(C^{\imath \jmath}\right)\right]:(-\Lambda, \Lambda) \times B_{\vartheta}(0) \rightarrow F_{0}^{*}, \quad\left(\lambda,\left(U^{i \jmath}\right)\right) \rightarrow\left(C^{\imath \jmath}\right)$,

- $\left[\left(D^{\imath j}\right)\right]:(-\Lambda, \Lambda) \times B_{\vartheta}(0) \rightarrow F_{0}^{*}, \quad\left(\lambda,\left(U^{\imath j}\right)\right) \rightarrow\left(D^{i j}\right)$

$$
:=4 \lambda^{2}\left(U^{k l} U^{\imath j}{ }_{k l}+U^{\imath j} U_{, k l}^{k l}-2 U^{k(i}{ }_{, k l} U^{j) l}\right),
$$

are of class $C^{\omega}\left((-\Lambda, \Lambda) \mapsto B_{\vartheta}(0), F_{0}^{*}\right)$, where $A^{i \jmath}, B^{\imath \jmath}, C^{\imath j}$ are defined in (2.2). Furthermore, it holds that

$$
\begin{aligned}
& D_{\left(U^{\imath j}\right)}\left[\left(A^{i j}\right)\left(0,\left(U^{i \jmath}\right)\right]\left(\delta U^{\imath \jmath}\right)=\delta U^{00, j} U^{00, i}+\delta U^{00, \imath} U^{00, \jmath}-\left[\bar{g}^{\imath j}\right]_{\lambda=0} \delta U^{00, a} U^{00}{ }_{, a}\right. \\
& D_{\left(U^{i j}\right)}\left[\left(B^{i \jmath}\right)\left(0,\left(U^{\imath j}\right)\right]\left(\delta U^{\imath \jmath}\right)=0,\right. \\
& D_{\left(U^{i \jmath}\right)}\left[\left(C^{i j}\right)\left(0,\left(U^{i j}\right)\right]\left(\delta U^{i \jmath}\right)=0,\right. \\
& D_{\left(U^{i \jmath}\right)}\left[\left(D^{\imath \jmath}\right)\left(0,\left(U^{\imath \jmath}\right)\right]\left(\delta U^{i j}\right)=0 .\right.
\end{aligned}
$$

Thus, the map $F^{i j}-\Delta U^{\imath \jmath}=A^{i j}+B^{i j}+C^{\imath j}+D^{\imath \jmath}$ is of class $C^{\omega}$.

Proposition $3.11,3.10$, and the product rule yield the existence of a $\Lambda$, such that

$$
\begin{aligned}
& {\left[4 \pi G|d| T^{i j}\right]:\left((-\Lambda, \Lambda) \times(-\Omega, \Omega) \times B_{\vartheta}(0) \times B_{\Theta}(0) \times B_{\Theta}(0)\right) \rightarrow F_{0}^{*}} \\
& \quad\left(\lambda, \omega,\left(U^{i \jmath}\right), \varrho, p\right) \rightarrow 4 \pi G|d| T^{i j}
\end{aligned}
$$

is of class $C^{\omega}$.

Finally, with (3.1) we see that the map $\Phi^{i \jmath}:\left(\lambda, \omega,\left(U^{\imath \jmath}\right), \varrho, p\right) \rightarrow F_{2}^{*}$,

$$
\begin{aligned}
& \left(\lambda, \omega,\left(U^{i j}\right), \varrho, p\right) \rightarrow \Phi^{\imath j}\left(\lambda, \omega,\left(U^{i \jmath}\right), \varrho, p\right) \\
& \quad:=U^{\imath \jmath}-\frac{-1}{4 \pi} \int_{\mathbb{R}^{3}} \frac{4 \pi G\left|d\left(x^{\prime}\right)\right| T^{\imath j}\left(x^{\prime}\right)-\left[F^{\imath j}\left(x^{\prime}\right)-\Delta U^{\imath \jmath}\left(x^{\prime}\right)\right]}{\left|x-x^{\prime}\right|} d^{3} x^{\prime}
\end{aligned}
$$

is differentiable. Calculation of the partial derivative with respect to $\left(U^{i j}\right)$ leads to

$$
\begin{aligned}
& D_{\left(U^{i \jmath}\right)}\left[\Phi^{i j}\left(0, \omega,\left(U^{\imath \jmath}\right), \varrho, p\right)\right]\left(\delta U^{i \jmath}\right) \\
& \quad=\delta U^{i j}-\frac{-1}{4 \pi} \int_{\mathbb{R}^{3}} \frac{\delta U^{00, j} U^{00, \imath}+\delta U^{00, i} U^{00, j}-\left[\bar{g}^{i j}\right]_{\lambda=0} \delta U^{00, a} U^{00}{ }_{a}}{\left|x-x^{\prime}\right|} d^{3} x^{\prime} .
\end{aligned}
$$

We can easily see that

$$
D_{\left(U^{\imath \jmath}\right)}\left[\Phi\left(0, \omega,\left(U^{\imath j}\right), \varrho, p\right)\right]: F_{2}^{*} \rightarrow F_{2}^{*}
$$

is bijective. 
Section 4.1 shows that for all $\varrho, p \in B_{\Theta}(0), \omega \in[-\Omega, \Omega]$ there is a solution $\left(\stackrel{N}{U^{\imath \jmath}}\right) \in B_{\vartheta / 2}(0)$ of the equations $\Phi^{\imath j}\left(0, \omega,\left({ }^{\imath} U^{\imath \jmath}\right), \varrho, p\right)=0$. Thus, the proposition follows by the implicit function Theorem 3.8 .

From $\Phi^{00}\left(\lambda, \omega,\left(U^{i j}\right)(\lambda, \omega, \varrho, p), \varrho, p\right)=0$ and the chain rule, it follows that for all $\delta \varrho \in F_{0}$,

$$
\begin{aligned}
0= & D_{\varrho}\left[\Phi^{00}\left(\lambda, \omega,\left(U^{i j}\right)(\lambda, \omega, \varrho, p), \varrho, p\right)\right] \delta \varrho \\
& +D_{\left(U^{\imath \jmath}\right)}\left[\Phi^{00}\left(\lambda, \omega,\left(U^{\imath j}\right)(\lambda, \omega, \varrho, p), \varrho, p\right)\right] D_{\varrho}\left[\left(U^{i j}\right)(\lambda, \omega, \varrho, p)\right] \delta \varrho .
\end{aligned}
$$

Since $U^{00,0}=0$ and $\left[g_{0}^{00}\right]_{\lambda=0}=0$, Eq. (4.5) leads to

$$
D_{\left(U^{\imath j}\right)}\left[\Phi^{00}\left(0, \omega,\left(U^{i j}\right), \varrho, p\right)\right]\left(\delta U^{\imath \jmath}\right)=\delta U^{00} .
$$

Because the energy-momentum tensor $T^{i j}$ and the functions $\Phi^{i j}$ depend linearly on $\varrho$, it holds that

$$
D_{\varrho}\left[\Phi^{00}\left(0, \omega,\left(U^{\imath \jmath}\right), \varrho, p\right)\right] \delta \varrho=\frac{1}{4 \pi} \int_{\mathbb{R}^{3}} \frac{4 \pi G \delta \varrho\left(x^{\prime}\right)}{\left|x-x^{\prime}\right|} d^{3} x^{\prime} .
$$

Insertion of this equation and of Eq. (4.7) into (4.6) leads to

$$
D_{\varrho}\left[\left(U^{i \jmath}\right)(0, \omega, \varrho, p)\right] \delta \varrho=-G \int_{\mathbb{R}^{3}} \frac{\delta \varrho\left(x^{\prime}\right)}{\left|x-x^{\prime}\right|} d^{3} x^{\prime} .
$$

With a similar calculation it can be shown that

$$
D_{p}\left[\left(U^{i j}\right)(0, \omega, \varrho, p)\right] \delta \varrho=0 .
$$

At the end of this section, we make use of the fact that the functions $\varrho, p$, and $\left(U^{\imath \jmath}\right)$ can be defined on $\mathbb{R}^{4}$. They depend trivially on the coordinate $x^{0}$. The matrix

$$
S_{\varphi}:=\left(\begin{array}{cccc}
1 & 0 & 0 & 0 \\
0 & \cos \varphi & -\sin \varphi & 0 \\
0 & \sin \varphi & \cos \varphi & 0 \\
0 & 0 & 0 & 1
\end{array}\right)
$$

represents a spacelike rotation around the $x^{3}$-axis with angle $\varphi$, and

$$
t:=\left(\begin{array}{cccc}
1 & 0 & 0 & 0 \\
0 & 1 & 0 & 0 \\
0 & 0 & 1 & 0 \\
0 & 0 & 0 & -1
\end{array}\right)
$$

represents a reflection at the plane $x^{3}=0$. Assume that the matter is symmetric relative to the $x^{3}$-axis and relative to the plane $x^{3}=0$, i.e., for all $\varphi$, the density $\varrho$ and the pressure $p$ fulfill

$$
\varrho\left(x^{\imath}\right)=\varrho\left(S_{\varphi \jmath}^{\imath} x^{\jmath}\right)=\varrho\left(t_{j}^{i} x^{\jmath}\right), \quad p\left(x^{\imath}\right)=p\left(S_{\varphi j}^{\imath} x^{j}\right)=p\left(t_{\jmath}^{i} x^{j}\right),
$$

respectively. Then, some calculations show, that for any solution $\left(U^{i j}\right)$ of the reduced field equations, the functions

$$
\tilde{U}^{\imath \jmath}\left(x^{\imath}\right)=S_{-\varphi a}^{i} S_{-\varphi a}^{\jmath} U^{a b}\left(S_{\varphi j}^{\imath} x^{\jmath}\right)
$$


and

$$
\hat{U}^{\imath \jmath}\left(x^{\imath}\right)=t_{a}^{\imath} t_{b}^{\jmath} U^{a b}\left(t_{\jmath}^{\imath} x^{\jmath}\right)
$$

also solve the reduced field equations. Due to Proposition 4.2, the solution of the reduced field equations is unique. Thus, the solution of the reduced field equations fulfills

$$
\begin{aligned}
& U^{\imath \jmath}\left(x^{\imath}\right)=S_{-\varphi a}^{\imath} S_{-\varphi a}^{\jmath} U^{i \jmath}\left(S \varphi_{\jmath}^{\imath} x^{\jmath}\right), \\
& U^{\imath \jmath}\left(x^{\imath}\right)=t_{a}^{\imath} t_{b}^{\jmath} U^{a b}\left(t_{\jmath}^{\imath} x^{\jmath}\right) .
\end{aligned}
$$

Equation (4.11) shows that any solution of the reduced field equations has the property

$$
\bar{g}_{\imath j}\left(x^{\imath}\right)=t_{\imath}^{a} t_{\jmath}^{b} \bar{g}_{a b}\left(t_{\jmath}^{\imath} x^{\jmath}\right) .
$$

With Eq. (4.10), it follows that the Lie derivative into the direction $\left(0,-x^{2}, x^{1}, 0\right)$ of the metric defined by $\left(U^{\imath \jmath}\right)$ vanishes. Thus, $\partial_{\varphi}=\left(0,-x^{2}, x^{1}, 0\right)$ is a Killing vector. Because the functions $\left(U^{i j}\right)$ are independent of the coordinate $x^{0}, \partial_{t}=(1,0,0,0)$ is also a Killing vector. Finally,

$$
\left(1,-\omega x^{2}, \omega x^{1}, 0\right)
$$

is a Killing vector of the metric, defined by the solution of the reduced field equations.

\section{Solution of Euler's Equation and of the Reduced Field Equations}

\subsection{Euler's Equation}

We assume that a set of equations of state $p_{\lambda}(\varrho)$, depending on $\lambda$, is given. According to some restrictions, presented in the next section, these equations of state fulfill $p_{\lambda} \geq 0, \partial_{\varrho} p_{\lambda} \geq 0$. Integration shows that Euler's equation (2.7) of an ideal fluid, rigidly rotating with angular velocity $\omega$, is equivalent to

$$
A_{\lambda}(\varrho(x))+\frac{1}{2 \lambda} \ln \left(u^{-2}(x)\right)-A_{\lambda}(\varrho(0))-\frac{1}{2 \lambda} \ln \left(u^{-2}(0)\right)=0,
$$

where the points $0, x$ lie within the star and

$$
\begin{aligned}
A_{\lambda}(\varrho) & =\int_{0}^{\varrho} \frac{\partial_{s} p_{\lambda}(s)}{s+\lambda p_{\lambda}(s)} d s, \\
u^{-2} & =-\sqrt{|d|} \bar{g}_{\imath \jmath} v^{2} v^{\jmath}, \\
v & =\left(1,-\omega \xi x^{2}, \omega \xi x^{1}, 0\right) .
\end{aligned}
$$

The function $\xi$, defined in (3.4), fulfills $\xi(x)=1$ for all $x$ with $|x| \leq R_{0}$. Obviously, the radius has to be chosen such that the whole matter lies in the Ball $B_{R_{0}}(0)$. The Newtonian limit $\lambda \rightarrow 0$ in Eq. (5.1) yields

$$
0=A_{0}(\varrho(x))+\stackrel{N}{U}^{00}(x)-\frac{\xi^{2} \omega^{2}}{2}\left[\left(x^{1}\right)^{2}+\left(x^{2}\right)^{2}\right]-A_{0}(\varrho(0))-\stackrel{N}{U}^{00}(0),
$$


where $\stackrel{N}{U}^{00}$ denotes the Newtonian gravitational potential (4.2). Equation (5.3) is Euler's equation of an ideal fluid, rigidly rotating with angular velocity $\omega$, in Newtonian fluid mechanics.

Assume that $\varrho_{0}: T \rightarrow \mathbb{R}^{+}$is a solution of Eq. (5.3) with angular velocity $\omega_{0}$. Here, $T$ denotes a smooth and compact domain. The coordinate system is chosen such that the star rigidly rotates around the $x^{3}$-axis. The solution $\varrho$ is rotational symmetric relative to this axis, and symmetric relative to reflections at the plane $x^{3}=0$. In $[12,13]$ it is shown that any solution of Euler's equation, representing a Newtonian star in thermal equilibrium, is rotational symmetric relative to the rotation axis, and symmetric relative to a plane, orthogonal to this axis. With the definition

$$
A_{0}\left(\varrho_{0}(x)\right):=-N^{N} 00(x)+\frac{\omega_{0}^{2} \xi^{2}}{2}\left[\left(x^{1}\right)^{2}+\left(x^{2}\right)^{2}\right]+A_{0}\left(\varrho_{0}(0)\right)+\stackrel{N}{U}^{00}(0)
$$

we extend the function $A_{0}\left(\varrho_{0}(x)\right)$ to points $x \notin T$.

Since the force, resulting from pressure, is directed towards the exterior of the star, for all $x$ on the surface of the star the sum of gravitational and centrifugal force

$$
-\nabla\left[\stackrel{N}{U}^{00}(x)-\frac{\xi^{2} \omega_{0}^{2}}{2}\left[\left(x^{1}\right)^{2}+\left(x^{2}\right)^{2}\right]=\nabla A_{0}\left(\varrho_{0}(x)\right)\right.
$$

is directed towards the interor. Let $n(x)$ denote the normal vector on the surface $\partial T$ at the point $x$ with $|n(x)|=1$, directed towards the exterior. Then, there exists a constant $\psi>0$, such that for all $x \in \partial T$ it holds that

$$
-n(x) \nabla A_{0}\left(\varrho_{0}(x)\right) \geq 2 \psi \text {. }
$$

Since the functions $\stackrel{N}{U}^{00}{ }_{, i}(x)-\frac{\xi^{2} \omega^{2}}{2} \partial_{x^{2}}\left[\left(x^{1}\right)^{2}+\left(x^{2}\right)^{2}\right]$ are continuous for all $\stackrel{N}{U}^{00} \in F_{2}$, there is an $\varepsilon$, such that for all $x \in \partial T$, and all $t \in[-\varepsilon, \varepsilon]$ it holds that

$$
-n(x) \nabla A_{0}\left(\varrho_{0}(x+t n(x))\right) \geq \psi,
$$

i.e. in the whole shell of size $\varepsilon$, the sum of gravitational and centrifugal force into direction $-n$ is greater than $\psi$. Let

$$
T_{\varepsilon}:=\left\{x^{\prime}=x+\operatorname{tn}(x) ; x \in \partial T,|t| \leq \varepsilon\right\}
$$

denote this shell. We choose $\varepsilon$ such that for all $x, \tilde{x} \in \partial T$, and all $t, \tilde{t} \in[-\varepsilon, \varepsilon]$ the equation

$$
x+\operatorname{tn}(x)=\tilde{x}+\tilde{t} n(\tilde{x})
$$

has the unique solution $x=\tilde{x}, t=\tilde{t}$. Consequently, any smooth function $t: \partial T \rightarrow$ $[-\varepsilon, \varepsilon]$ uniquely defines a surface of a new body by $\partial T \rightarrow \mathbb{R}^{3}, x \rightarrow x+t(x) n(x)$.

\subsection{Restrictions to the Equations of State}

From a given tensor density $\left(U^{i j}\right)$, that defines a function $u^{-2}$, we calculate the density $\varrho$ and the pressure $p$ with Euler's equation. Since the implicit function Theorem 3.8 will be used to prove the existence of solutions $\left(\lambda, \varrho, p\left(U^{i j}\right)\right)$ of Euler's equation and the reduced field equations, this operation must be differentiable. This leads to some restrictions to the equations of state. 
The equations of state $p_{\lambda}(\varrho)$ are assumed to fulfill $p_{\lambda} \geq 0, \partial_{\varrho} p_{\lambda} \geq 0$, and $p_{\lambda}(0)=0$. Because the surface of the star is defined by $p_{\lambda}=0$, it follows that the density $\varrho$ also vanishes on the surface of the star.

Let $A_{\max }, \tau$, and $\Lambda$ be constants, such that for all $0 \leq \lambda \leq \Lambda$, all $x \in T$, and all $0 \leq t \leq \tau$ it holds that

$$
A_{\lambda}\left(\varrho_{0}(x)\right)+t \leq A_{\max }
$$

The constant $A_{\max }$ must be chosen in a way that for all $0 \leq \lambda \leq \Lambda$ the functions

$$
\begin{aligned}
F_{\lambda}(y) & := \begin{cases}A_{\lambda}^{-1}(y) & \text { for } y \in\left[0, A_{\max }\right] \\
0 & \text { for } y \leq 0\end{cases} \\
G_{\lambda}(y) & :=\left\{\begin{array}{ll}
p_{\lambda} \circ A_{\lambda}^{-1}(y) & \text { for } y \in\left[0, A_{\max }\right] \\
0 & \text { for } y \leq 0
\end{array}=p_{\lambda}\left(F_{\lambda}(y)\right)\right.
\end{aligned}
$$

exist, and are continuously differentiable with respect to $y$. The functions $F_{\lambda}(y)$, $G_{\lambda}(y)$, and their derivatives $F_{\lambda}^{\prime}(y), G_{\lambda}^{\prime}(y)$ are uniformly continuous for $0 \leq \lambda \leq \Lambda$, and $-\infty<y \leq A_{\max }$. Furthermore, for all $\varrho, \lambda_{0}$ there exists a constant $C$, such that the estimate

$$
\left|A_{\lambda}(\varrho)-A_{\lambda_{0}}(\varrho)\right| \leq C\left|\lambda-\lambda_{0}\right|
$$

is fulfilled.

Example. We discuss these restrictions in the case of a polytropic equation of state

$$
p(\varrho)=C \varrho^{\gamma} \text { with } \quad \gamma>1
$$

For arbitrary $A_{\max }>0$ we have

$$
\begin{aligned}
& A_{\lambda}(\varrho)=\frac{\gamma}{\lambda(\gamma-1)} \ln \left(1+\lambda C \varrho^{\gamma-1}\right), \\
& F_{\lambda}(\varrho)= \begin{cases}{\left[\frac{1}{C \lambda}\left(e^{\lambda \frac{\gamma-1}{\gamma} y}-1\right]^{\frac{1}{\gamma-1}}\right.} & \text { for } y \in\left[0, A_{\max }\right], \\
0 & \text { for } y \leq 0\end{cases} \\
& G_{\lambda}(\varrho)= \begin{cases}C\left[\frac{1}{C \lambda}\left(e^{\lambda \frac{\gamma-1}{\gamma} y}-1\right]^{\frac{1}{\gamma-1}}\right. & \text { for } y \in\left[0, A_{\max }\right] . \\
0 & \text { for } y \leq 0\end{cases}
\end{aligned}
$$

From these equations, it can easily be seen that the conditions of this section are fulfilled if the functions $f(x):=x^{\frac{1}{\gamma-1}}$ and $g(x):=x^{\frac{1}{\gamma-1}}$ are continuously differentiable in $\mathbb{R}^{+}$, and

$$
\lim _{x \rightarrow 0} \partial_{x} f(x)=\lim _{x \rightarrow 0} \partial_{x} g(x)=0 .
$$

This yields the restriction $\frac{1}{\gamma-1}>1$, such that the polytropic index $\gamma$ has to fulfill

$$
1<\gamma<2
$$




\subsection{Solution of Euler's Equation and of the Reduced Field Equations}

Definition 5.1. Let $p \in \mathbb{R}$ be the number, that is introduced in Defintion 3.9. The density $\varrho$ and the pressure $p$ lie in the Banach space

$L_{S}^{p}\left(T \cup T_{\varepsilon}\right):=\left\{\begin{array}{l|l}f \in L^{p}\left(T \cup T_{\varepsilon}\right) & \begin{array}{l}f \text { is symmetric relative to the } x^{3} \text {-axis, a.e. } \\ f \text { is symmetric relative to the plane } x^{3}=0 \text {, a.e. }\end{array}\end{array}\right\}$ with the norm $\|\cdot\|_{p}$. Note that $T \cup T_{\varepsilon}$ is compact.

Obviously, the radius $R_{0}$, used to define the function $\xi$ Eq. (3.4), must be chosen such that $T \cup T_{\varepsilon} \subset B_{R_{0}}(0)$.

Proposition 5.2. Let $\varrho_{0}$ be the solution of Euler's equation in Newtonian fluid mechanics, introduced in Sect. 5.1. Then, there exist $\Lambda>0, \Omega>0$, and $\delta>0$, such that for all $0 \leq \lambda \leq \Lambda$ the condition

$$
f_{\lambda}(x):=-\frac{1}{2 \lambda} \ln \left(u_{\varrho, p}^{-2}(x)\right)+\frac{1}{2 \lambda} \ln \left(u_{\varrho, p}^{-2}(0)\right)+A_{\lambda}\left(\varrho_{0}(0)\right)=0
$$

defines a closed, and smooth surface in the shell $T_{\varepsilon}$. Here, the function $u_{\varrho, p}^{-2}$ is defined by the solution $\left[\left(U^{i j}\right)\right](\lambda, \omega, \varrho, p)$ of the reduced field equations (Proposition 4.2), and

$$
\left\|\varrho-\varrho_{0}\right\|_{p}, \quad\left\|p-p_{0}\left(\varrho_{0}\right)\right\|_{p}<\delta, \quad\left|\omega-\omega_{0}\right| \leq \Omega .
$$

For all points $x \in T \cup T_{\varepsilon}$ outside the domain surrounded by this surface, it holds that $f_{\lambda}(x)<0$.

Furthermore, for all $\lambda \leq \Lambda$, the functions

$$
\begin{aligned}
T:[0, \Lambda] \times & {\left[\omega_{0}-\Omega, \omega_{0}+\Omega\right] \times B_{\delta}\left(\varrho_{0}\right) \subset L_{S}^{p}\left(T \cup T_{\varepsilon}\right) } \\
\times & B_{\delta}\left(p_{0}\left(\varrho_{0}\right)\right) \subset L_{S}^{p}\left(T \cup T_{\varepsilon}\right) \rightarrow L_{S}^{p}\left(T \cup T_{\varepsilon}\right) \\
& (\lambda, \omega, \varrho, p) \rightarrow F_{\lambda}\left(-\frac{1}{2 \lambda} \ln \left(u_{\varrho, p}^{-2}(x)\right)+\frac{1}{2 \lambda} \ln \left(u_{\varrho, p}^{-2}(0)\right)+A_{\lambda}\left(\varrho_{0}(0)\right)\right)
\end{aligned}
$$

and

$$
\begin{aligned}
S:[0, \Lambda] \times & {\left[\omega_{0}-\Omega, \omega_{0}+\Omega\right] \times B_{\delta}\left(\varrho_{0}\right) \subset L_{S}^{p}\left(T \cup T_{\varepsilon}\right) } \\
\times & B_{\delta}\left(p_{0}\left(\varrho_{0}\right)\right) \subset L_{S}^{p}\left(T \cup T_{\varepsilon}\right) \rightarrow L_{S}^{p}\left(T \cup T_{\varepsilon}\right), \\
& (\lambda, \omega, \varrho, p) \rightarrow G_{\lambda}\left(-\frac{1}{2 \lambda} \ln \left(u_{\varrho, p}^{-2}(x)\right)+\frac{1}{2 \lambda} \ln \left(u_{\varrho, p}^{-2}(0)\right)+A_{\lambda}\left(\varrho_{0}(0)\right)\right)
\end{aligned}
$$

can be defined, and have the property

$$
[T(\lambda, \omega, \varrho, p)](x)=[S(\lambda, \omega, \varrho, p)](x)=0
$$

for $x \in \partial\left(T \cup T_{\varepsilon}\right)$. The functions $F_{\lambda}, G_{\lambda}$ were introduced in Sect. 5.2.

Proof. First, we take any $\Omega>0, \delta>0$, and restrict $\Lambda$ such that Propositions 4.2, 3.10, 3.11 hold, and the equations of state have the properties of Sect. 5.2.

Due to Proposition 3.11, 4.2, and the chain rule, the function

$$
\begin{aligned}
t:(-\Lambda, \Lambda) \times(-\Omega, \Omega) \times & B_{\delta}\left(\varrho_{0}\right) \subset L^{p}\left(T \cup T_{\varepsilon}\right) \\
\times & B_{\delta}\left(p_{0}\left(\varrho_{0}\right)\right) \subset L^{p}\left(T \cup T_{\varepsilon}\right) \rightarrow F_{2}, \\
& (\lambda, \omega, \varrho, p) \rightarrow\left[\frac{1}{2 \lambda} \ln \left(u^{-2}\right)\right]\left(\lambda, \omega,\left[\left(U^{i j}\right)\right](\lambda, \omega, \varrho, p)\right)
\end{aligned}
$$


is continuously differentiable. It should be emphasized that continuous embeddings are of class $C^{\omega}$, because they are linear. Thus, the continuous embedding $L^{p}\left(T \cup T_{\varepsilon}\right) \rightarrow F_{0}$ is of class $C^{\omega}\left(L^{p}\left(T \cup T_{\varepsilon}\right), F_{0}\right)$, such that Proposition 4.2 can also be used if $\varrho, p \in L_{S}^{p}\left(T \cup T_{\varepsilon}\right)$, rather than $\varrho, p \in F_{0}$.

With (4.2) and (3.6) it follows

$$
\begin{aligned}
{[t(0, \omega, \varrho, p)](x) } & =-\xi^{2} \frac{\omega^{2}}{2}\left(\left(x^{1}\right)^{2}+\left(x^{2}\right)^{2}\right)+\left[U^{00}(0, \omega, \varrho, p)\right](x) \\
& =-\xi^{2} \frac{\omega^{2}}{2}\left(\left(x^{1}\right)^{2}+\left(x^{2}\right)^{2}\right)-G \int_{T \cup T_{\varepsilon}} \frac{\varrho\left(x^{\prime}\right)}{\left|x-x^{\prime}\right|} d^{3} x^{\prime}
\end{aligned}
$$

Because $t$ is continuously differentiable, Taylor's theorem 3.7 leads to the existence of a constant $C_{1}$, such that for all $\varrho \in B_{\delta}\left(\varrho_{0}\right)$, all $p \in B_{\delta}\left(p_{0}\left(\varrho_{0}\right)\right)$, and all $\left|\omega-\omega_{0}\right|<\Omega$ it holds that

$$
\|t(\lambda, \omega, \varrho, p)-t(0, \omega, \varrho, p)\|_{F_{2}} \leq C_{1} \lambda .
$$

Since $\varrho_{0}$ is a solution of Euler's equation in Newtonian fluid mechanics, Eqs. (5.4), and (4.2) yield for all $x \in T \cup T_{\varepsilon}$,

$$
\begin{gathered}
-\left[U^{00}(0, \omega, \varrho, p)\right](x)+\frac{\xi^{2} \omega^{2}}{2}\left[\left(x^{1}\right)^{2}+\left(x^{2}\right)^{2}\right]+\left[U^{00}(0, \omega, \varrho, p)\right](0)+A_{0}\left(\varrho_{0}(0)\right) \\
=A_{0}\left(\varrho_{0}(x)\right)-U_{\varrho-\varrho_{0}}(x)+\frac{\xi^{2}\left[\omega^{2}-\omega_{0}^{2}\right]}{2}\left[\left(x^{1}\right)^{2}+\left(x^{2}\right)^{2}\right]+U_{\varrho-\varrho_{0}}(0),
\end{gathered}
$$

where for any $f \in L^{p}\left(T \cup T_{\varepsilon}\right)$ the function $U_{f}$ is defined by

$$
U_{f}(x):=-G \int_{T_{\varepsilon} \cup T} \frac{f\left(x^{\prime}\right)}{\left|x-x^{\prime}\right|} d^{3} x^{\prime} .
$$

This equation, the continuity of the embedding $L^{p}\left(T \cup T_{\varepsilon}\right) \rightarrow F_{0}$, and (3.1) lead to the existence of a constant $C_{2}$ such that

$$
\begin{aligned}
& \|-\left[U^{00}(0, \omega, \varrho, p)\right](x)+\frac{\xi^{2} \omega^{2}}{2}\left[\left(x^{1}\right)^{2}+\left(x^{2}\right)^{2}\right]+\left[U^{00}(0, \omega, \varrho, p)\right](0)+A_{0}\left(\varrho_{0}(0)\right) \\
& -A_{0}\left(\varrho_{0}(x)\right) \|_{F_{2}} \leq C_{2}(\Omega+\delta)
\end{aligned}
$$

With

$$
\begin{aligned}
f_{\lambda}(x) & -A_{0}\left(\varrho_{0}(x)\right) \\
= & (-[t(\lambda, \omega, \varrho, p)](x)+[t(0, \omega, \varrho, p)](x))+\left(-\left[U^{00}(0, \omega, \varrho, p)\right](x)-A_{0}\left(\varrho_{0}(x)\right)\right. \\
& \left.+\frac{\xi^{2} \omega^{2}}{2}\left[\left(x^{1}\right)^{2}+\left(x^{2}\right)^{2}\right]+\left[U^{00}(0, \omega, \varrho, p)\right](0)+A_{0}\left(\varrho_{0}(0)\right)\right) \\
& \left.+([t(\lambda, \omega, \varrho, p)](0)-[t(0, \omega, \varrho, p)](0))+A_{\lambda}\left(\varrho_{0}(0)\right)-A_{0}\left(\varrho_{0}(0)\right)\right),
\end{aligned}
$$

(5.11), (5.12), the continuity of the embedding $F_{2} \rightarrow C^{1, \nu}\left(\overline{\mathbb{R}}^{3}\right)$, and (5.9), there follows the existence of a constant $C$ such that

$$
\begin{aligned}
\left|f_{\lambda}(x)-A_{0}\left(\varrho_{0}(x)\right)\right| & \leq C(\Omega+\delta+\Lambda), \\
\left|\nabla\left[f_{\lambda}(x)-A_{0}\left(\varrho_{0}(x)\right)\right]\right| & \leq C(\Omega+\delta+\Lambda) .
\end{aligned}
$$


Since $\varrho_{0}\left(x_{0}\right)=0$ for all $x_{0} \in \partial T$, it holds that $A_{0}\left(\varrho_{0}\left(x_{0}\right)\right)=0$. This equation and estimate (5.13) yield

$$
\left|f_{\lambda}\left(x_{0}\right)\right| \leq C(\Omega+\delta+\Lambda)
$$

If we choose $\Omega, \delta, \Lambda<\frac{\psi}{6 C}$, then (5.5) and (5.13) lead to

$$
-n\left(x_{0}\right) \nabla f_{\lambda}\left(x_{0}+\operatorname{tn}\left(x_{0}\right)\right)>\frac{\psi}{2}
$$

for all $t \in[-\varepsilon, \varepsilon]$. Thus, the function $g(t):=f_{\lambda}\left(x_{0}+\operatorname{tn}\left(x_{0}\right)\right)$ fulfills $|g(0)| \leq$ $C(\Omega+\delta+\Lambda)$, and in $[-\varepsilon, \varepsilon]$ it has a negative derivative that is greater than $\frac{\psi}{2}$. If
we choose

$$
\Omega, \delta, \Lambda<\frac{\psi \varepsilon}{6 C}
$$

then $g(t)$ has a unique zero in $(-\varepsilon, \varepsilon)$. Consequently, for all $x \in \partial T$ there is a unique zero of the function $f_{\lambda}$ on the line, defined by $x_{0}$ and the direction $n\left(x_{0}\right)$. Due to the assumtpion, there is no intersection point of the lines $x_{0}+\operatorname{tn}\left(x_{0}\right)$ and $\tilde{x}_{0}+\operatorname{tn}\left(\tilde{x}_{0}\right)$ if $t \in[-\varepsilon, \varepsilon]$ and $x_{0} \neq \tilde{x}_{0}$. Furthermore, the function $f_{\lambda}$ is continuously differentiable. Thus, the condition $f_{\lambda}(x)=0$ uniquely defines a closed, smooth surface in the shell $T_{\varepsilon}$. In addition, it holds that $f_{\lambda}(x)<0$ for all $x$ outside the domain that is surrounded by this surface.

If we restrict $\Omega, \delta$, and $\Lambda$ such that

$$
\left|f_{\lambda}(x)-A_{0}\left(\varrho_{0}(x)\right)\right| \leq \tau,
$$

condition $\left|f_{\lambda}(x)\right| \leq A_{\max }$ is fulfilled (compare (5.7), and the functions $F_{\lambda}\left(f_{\lambda}(x)\right.$ ), $G_{\lambda}\left(f_{\lambda}(x)\right)$ are defined for all $x \in T_{\varepsilon} \cup T$. These functions are continuous, and it holds that

$$
[T(\varrho, p, \omega, \lambda)](x)=[S(\varrho, p, \omega, \lambda)](x)=0
$$

for all $x \in \partial\left(T \cup T_{\varepsilon}\right)$. This equation immediately follows with $f_{\lambda}\left(x_{0}\right)<0$ for $x \in \partial\left(T \cup T_{\varepsilon}\right)$, and $F_{\lambda}(y)=G_{\lambda}(y)=0$ for $y<0$.

In order to prove that the functions $T$, and $S$ map into $L_{S}^{p}\left(T \cup T_{\varepsilon}\right)$, it remains to be shown that the function $f_{\lambda}$ is symmetric relative to the $x^{3}$-axis and relative to the plane $x^{3}=0$. Equation (4.12) and $v^{i}\left(x^{1}, x^{2}, x^{3}\right)=v^{i}\left(x^{1}, x^{2},-x^{3}\right)$ lead to $u_{\varrho, p}^{-2}\left(x^{1}, x^{2}, x^{3}\right)=u_{\varrho, p}^{-2}\left(x^{1}, x^{2},-x^{3}\right)$, such that $f_{\lambda}\left(x^{1}, x^{2}, x^{3}\right)=f_{\lambda}\left(x^{1}, x^{2},-x^{3}\right)$. At the end of Sect. 4.2 it was shown that $v=\left(1,-\omega x^{2}, \omega x^{1}, 0\right)=\partial_{t}+\omega \partial_{\varphi}$ is a Killing vector. Since all functions are independent of the coordinate $x^{0}$, it follows that

$$
\begin{aligned}
\partial_{\varphi} u_{\varrho, p}^{-2} & =v^{\imath} \nabla_{\imath} u_{\varrho, p}^{-2} \\
& =v^{i} \nabla_{\imath}\left(-\lambda v^{j} v_{j}\right) \\
& =-2 \lambda v^{\imath} v^{\jmath} \nabla_{\imath} v_{\jmath} \\
& =0
\end{aligned}
$$

such that $\partial_{\varphi} f_{\lambda}=0$. Here, we must keep in mind that $\xi(x)=1$ for all $x \in T \cup$ $T_{\varepsilon}$. 
Proposition 5.3. Let $p_{\lambda}(\varrho)$ be a set of equations of state, that fulfills the restrictioms of Sect. 5.2. Assume that $\varrho_{0}(x)$ is the solution of Euler's equation in Newtonian fluid mechanics, introduced in Sect. 5.1. Additionally, it is assumed that the integral equation

$$
h_{0}(x)=F_{0}^{\prime}\left(A_{0}\left(\varrho_{0}(x)\right)\right) G \int_{T} h_{0}\left(x^{\prime}\right)\left(\frac{1}{\left|x-x^{\prime}\right|}-\frac{1}{\left|x^{\prime}\right|}\right) d^{3} x^{\prime}
$$

has the unique solution $h_{0}=0$ in

$$
C_{S}^{0}(T):=\left\{\begin{array}{l|l}
f \in C^{0}(T) & \begin{array}{l}
f \text { is symmetric relative to the } x^{3} \text {-axis } \\
f \text { is symmetric relative to the plane } x^{3}=0
\end{array}
\end{array}\right\} .
$$

Then, there exist constants $\Lambda>0, \Omega>0$, such that for all $0 \leq \lambda<\Lambda$ and all $\left|\omega-\omega_{0}\right|<\Omega$, there exists a solution $\left(\lambda, \omega, \varrho,\left(U^{i j}\right)\right)$ of Euler's equation (5.1) and of the reduced field equations (2.3).

Proof. According to Proposition 5.2, there exist constants $\Lambda, \Omega$, and $\delta$ such that for all $0 \leq \lambda \leq \Lambda$, and all $\left|\omega-\omega_{0}\right| \leq \Omega$ the function

$$
\begin{gathered}
\Psi_{\lambda, \omega}: B_{\delta}\left(\varrho_{0}\right) \subset L_{S}^{p}\left(T \cup T_{\varepsilon}\right) \times B_{\delta}\left(p_{0}\left(\varrho_{0}\right)\right) \subset L_{S}^{p}\left(T \cup T_{\varepsilon}\right) \rightarrow L_{S}^{p}\left(T \cup T_{\varepsilon}\right) \times L_{S}^{p}\left(T \cup T_{\varepsilon}\right), \\
\left(\begin{array}{l}
\varrho \\
p
\end{array}\right) \rightarrow\left(\begin{array}{l}
\varrho-T(\lambda, \omega, \varrho, p) \\
p-S(\lambda, \omega, \varrho, p)
\end{array}\right)
\end{gathered}
$$

can be defined. We can easily convince ourselves that the zeros of $\Psi_{\lambda, \omega}$ determine a solution of Euler's equation and of the reduced field equations. This solution represents a body, rigidly rotating with angular velocity $\omega$, and consisting of ideal fluid with equation of state $p_{\lambda}(\varrho)$. The matter of this solution completely lies in $T \cup T_{\varepsilon}$. Thus, we have to prove the existence of zeros of the function $\Psi_{\lambda, \omega}$ in the neighbourhood of the given zero $\Psi_{0, \omega_{0}}\left(\varrho_{0}, p_{0}\left(\varrho_{0}\right)\right)=0$. This can be done with the implicit function theorem 3.8.

Some straightforward calculations show, that for any Hölder constant $0<\nu<1$, and any constant $C$, the functions

$$
\begin{array}{ll}
t_{\lambda}: B_{M}(0) \subset C^{1, \nu}\left(T \cup T_{\varepsilon}\right) \rightarrow C^{0}\left(T \cup T_{\varepsilon}\right), & f(x) \rightarrow F_{\lambda}(f(x)-f(0)+C), \\
s_{\lambda}: B_{M}(0) \subset C^{1, \nu}\left(T \cup T_{\varepsilon}\right) \rightarrow C^{0}\left(T \cup T_{\varepsilon}\right), & f(x) \rightarrow G_{\lambda}(f(x)-f(0)+C)
\end{array}
$$

are of class $C^{1}\left(B_{M}(0), C^{0}\left(T \cup T_{\varepsilon}\right)\right)$. Here, $M$ must be restricted such that $\mid f(x)-$ $f(0)+C \mid<A_{\max }$. Furthermore, with the properties of $F_{\lambda}$ and $G_{\lambda}$, presented in Sect. 5.2, it can be seen that these funtions and their derivatives

$$
\begin{aligned}
& D\left[t_{\lambda}(f)\right]: C^{1, \nu}\left(T \cup T_{\varepsilon}\right) \rightarrow C^{0}\left(T \cup T_{\varepsilon}\right), \\
& \quad \delta f(x) \rightarrow F_{\lambda}^{\prime}(f(x)-f(0)+C) \cdot(\delta f(x)-\delta f(0)), \\
& D\left[s_{\lambda}(f)\right]: C^{1, \nu}\left(T \cup T_{\varepsilon}\right) \rightarrow C^{0}\left(T \cup T_{\varepsilon}\right), \\
& \quad \delta f(x) \rightarrow G_{\lambda}^{\prime}(f(x)-f(0)+C) \cdot(\delta f(x)-\delta f(0))
\end{aligned}
$$

depend continuously on $\lambda$.

Since the continuous embeddings $C^{0}\left(T \cup T_{\varepsilon}\right) \rightarrow L^{p}\left(T \cup T_{\varepsilon}\right), F_{2} \rightarrow C^{1, \nu}\left(\overline{\mathbb{R}}^{3}\right)$ are of class $C^{\omega}$, the chain rule, together with Propositions 3.11, 4.2, yields that the functions

$$
\begin{aligned}
& T(\lambda, \omega, \varrho, p)=t_{\lambda}\left(-[t(\lambda, \omega, \varrho, p)](x)+[t(\lambda, \omega, \varrho, p)](0)+A_{\lambda}\left(\varrho_{0}(0)\right)\right), \\
& S(\lambda, \omega, \varrho, p)=s_{\lambda}\left(-[t(\lambda, \omega, \varrho, p)](x)+[t(\lambda, \omega, \varrho, p)](0)+A_{\lambda}\left(\varrho_{0}(0)\right)\right)
\end{aligned}
$$


are continuously differentiable with respect to $\omega, \varrho$, and $p$. Here, $t$ is defined in (5.10). Thus, all functions $\Psi_{\lambda, \omega}$ are continuously differentiable, and $\Psi_{\lambda, \omega}$ and its derivative

$$
\begin{gathered}
D\left[\Psi_{\lambda, \omega}(\varrho, p)\right]: L_{S}^{p}\left(T \cup T_{\varepsilon}\right) \times L_{S}^{p}\left(T \cup T_{\varepsilon}\right) \rightarrow L_{S}^{p}\left(T \cup T_{\varepsilon}\right) \times L_{S}^{p}\left(T \cup T_{\varepsilon}\right), \\
\left(\begin{array}{c}
\delta \varrho \\
\delta p
\end{array}\right) \rightarrow\left(\begin{array}{cc}
1-D_{\varrho}[T(\lambda, \omega, \varrho, p)] & D_{p}[T(\lambda, \omega, \varrho, p)] \\
D_{\varrho}[S(\lambda, \omega, \varrho, p)] & \mathbb{1}-D_{p}[S(\lambda, \omega, \varrho, p)]
\end{array}\right)\left(\begin{array}{c}
\delta \varrho \\
\delta p
\end{array}\right)
\end{gathered}
$$

depend continuously on $\lambda$ and $\omega$. Consequently, the proposition follows by the implicit function theorem 3.8, if the operator $D\left[\Psi_{0, \omega_{0}}\left(\varrho_{0}, p_{0}\left(\varrho_{0}\right)\right)\right]$ is bijective.

With the chain rule, Proposition 3.11, and Eqs. (4.8), (4.9), (5.15), we see that

$$
D\left[\Psi_{0, \omega_{0}}\left(\varrho_{0}, p_{0}\left(\varrho_{0}\right)\right)\right]\left(\begin{array}{l}
\delta \varrho \\
\delta p
\end{array}\right)=\left(\begin{array}{cc}
\mathbb{1}-D_{\varrho}\left[T\left(0, \omega_{0}, \varrho_{0}, p_{0}\left(\varrho_{0}\right)\right)\right] & 0 \\
D_{\varrho}\left[S\left(0, \omega_{0}, \varrho_{0}, p_{0}\left(\varrho_{0}\right)\right)\right] & \mathbb{1}
\end{array}\right)\left(\begin{array}{l}
\delta \varrho \\
\delta p
\end{array}\right)
$$

where

$$
\begin{aligned}
& {\left[D_{\varrho}\left[T\left(0, \omega_{0}, \varrho_{0}, p_{0}\left(\varrho_{0}\right)\right)\right] \delta \varrho\right](x)} \\
& \quad=F_{0}^{\prime}\left(A_{0}\left(\varrho_{0}(x)\right)\right) G \int_{T \cup T_{\varepsilon}} \delta \varrho\left(x^{\prime}\right)\left(\frac{1}{\left|x-x^{\prime}\right|}-\frac{1}{\left|x^{\prime}\right|}\right) d^{3} x^{\prime} .
\end{aligned}
$$

$A_{0}\left(\varrho_{o}(x)\right)$ is defined in (5.4). It can easily be seen that $D\left[\Psi_{0, \omega_{0}}\left(\varrho_{0}, p_{0}\left(\varrho_{0}\right)\right)\right]$ is bijective if

$$
\mathbb{1}-D_{\varrho}\left[T\left(0, \omega_{0}, \varrho_{0}, p_{0}\left(\varrho_{0}\right)\right)\right]: L_{S}^{p}\left(T \cup T_{\varepsilon}\right) \rightarrow L_{S}^{p}\left(T \cup T_{\varepsilon}\right)
$$

is bijective.

Due to Sect. 3.3.1 the embeddings $L^{p}\left(T \cup T_{\varepsilon}\right) \rightarrow F_{0}, C^{1, \nu}\left(T \cup T_{\varepsilon}\right) \rightarrow L^{p}\left(T \cup T_{\varepsilon}\right)$ and the operator $\Delta^{-1}: F_{0} \rightarrow F_{2}$ are continuous, and the embedding $F_{2} \rightarrow C^{1, \nu}\left(T \cup T_{\varepsilon}\right)$ is compact. Since concatenation of continuous operators and a compact operator yields a compact operator, the map

$$
f \in L^{p}\left(T \cup T_{\varepsilon}\right) \rightarrow \int_{T \cup T_{\varepsilon}} f\left(x^{\prime}\right)\left(\frac{1}{\left|x-x^{\prime}\right|}-\frac{1}{\left|x^{\prime}\right|}\right) d^{3} x^{\prime} \in L^{p}\left(T \cup T_{\varepsilon}\right)
$$

is compact. Because the multiplication with the bounded function $F_{0}^{\prime}\left(A_{0}\left(\varrho_{0}(x)\right)\right)$ is continuous, the operator $D_{\varrho}\left[T\left(0, \omega_{0}, \varrho_{0}, p_{0}\left(\varrho_{0}\right)\right)\right]$ is compact. According to [10], it follows that $\mathbb{1}-D_{\varrho} T\left(0, \omega_{0}, \varrho_{0}, p_{0}\left(\varrho_{0}\right)\right)$ is bijective, if it is injective, i.e. if its kernel is trivial.

Since $A_{0}\left(\varrho_{0}\left(x_{0}\right)\right)<0$ for all $x_{0} \in\left(T \cup T_{\varepsilon}\right) \backslash T$, it follows that $F_{0}^{\prime}\left(A_{0}\left(\varrho_{0}\left(x_{0}\right)\right)\right)=0$. Furthermore, $\left[D_{\varrho} T\left(0, \omega_{0}, \varrho_{0}, p_{0}\left(\varrho_{0}\right)\right)\right] h_{0}$ is continuous for all $h_{0} \in L^{p}\left(T \cup T_{\varepsilon}\right)$. Thus, any function $h_{0} \in L_{S}^{p}\left(T \cup T_{\varepsilon}\right)$ that fulfills

$$
h_{0}=D_{\varrho} T\left(0, \omega_{0}, \varrho_{0}, p_{0}\left(\varrho_{0}\right)\right) h_{0}
$$

lies in the subspace

$$
C_{S}^{0}(T):=\left\{\begin{array}{l|l}
f \in C^{0}(T) & \begin{array}{l}
f \text { is symmetric relative to the } x^{3} \text {-axis } \\
f \text { is symmetric relative to the plane } x^{3}=0
\end{array}
\end{array}\right\},
$$

and the operator $D\left[\Psi_{0, \omega_{0}}\left(\varrho_{0}, p_{0}\left(\varrho_{0}\right)\right)\right]$ is bijective if Eq. (5.14) has the unique solution $h_{0}=0$ in $C_{S}^{0}(T)$. 


\section{The Harmonic Conditions}

In this section we show that for sufficiently small $\Lambda$ and all $\lambda \leq \Lambda$, a solution of the reduced field equations and the equations of motion (2.3) automatically fulfills the harmonic conditions

$$
g_{, \imath}^{\imath \jmath}=0, \quad U^{i \jmath}{ }_{, i}=0,
$$

such that Einstein's equation are fulfilled. Precisely, we prove

Proposition 6.1. Let $T \subset \mathbb{R}^{3}$ be a compact domain with a surface smooth enough that Gauß' theorem holds in T. Furthermore, let $n_{\imath}(i=1,2,3)$ be the normal vector that is orthogonal to the surface $\partial T$ and points towards the outside. Assume that $T^{i j}: T \in \mathbb{R}$ is a $\lambda$-depending set $(0 \leq \lambda \leq \Lambda)$ of continuously differentiable energy-momentum tensors, and $\left(U^{i j}\right) \in F_{2}^{*}$ a set of tensor densities such that the equations of motion and the reduced field equations (2.3) are fulfilled with the causality constant $\lambda$. If

- there exist constants $\Theta, \vartheta$, such that for all $0 \leq \lambda \leq \Lambda_{0}$ it holds that $\left\|\left(U^{i j}\right)\right\|_{F_{2}^{*}} \leq \vartheta$, $\left\|T^{i \jmath}\right\|_{C^{1}(T)} \leq \Theta$,

- the solution is stationary, i.e. $\left(U^{\imath \jmath}{ }_{, 0}\right)=\left(T^{\imath \jmath}{ }_{, 0}\right)=0_{3}$

- for all $x_{0} \in \partial T$ and all $j=0,1,2,3$ it holds that $\sum_{i=1} n_{\imath}\left(x_{0}\right) T^{\imath \jmath}\left(x_{0}\right)=0$,

then there is a constant $\Lambda>0$ such that for all $0 \leq \lambda \leq \Lambda$ and $j=0,1,2,3$ the harmonic conditions

$$
U^{\imath \jmath}{ }_{, i}=0
$$

are fulfilled, i.e. $\left(\lambda,\left(U^{i j}\right), T^{i j}\right)$ are solutions of Einstein's equations (2.3) with causality constant $\lambda$.

Remarks.

- It is not required that $\left(T^{\imath j}\right)$ can be extended continuously to $\mathbb{R}^{3}$.

- This proposition also holds if the support of $T^{i j}$ is unbounded and $\left(T^{\imath \jmath}\right),\left(T^{i \jmath}{ }_{, k}\right) \in$ $F_{0}^{*}$.

- This proposition can be generalized to piecewise continuously differentiably energymomentum tensors $\left(T^{\imath \jmath}\right)$. In this case, it has to be required that in every domain of continuity Gauß's theorem is applicable, and the conditions $\sum_{\imath=1}^{3} n_{\imath}\left(x_{0}\right) T^{\imath \jmath}\left(x_{0}\right)=0$
hold on all boundary-surfaces.

In order to prove this proposition, we need the following properties of the Christoffel symbols.

Proposition 6.2. Let $B_{\vartheta}(0)$ denote the ball with radius $\vartheta$ centered at the origin of $F_{2}^{*}$. Then, for any $\vartheta>0$ there is a $\Lambda>0$ such that the functions mapping the causality constant $\lambda$ and the tensor density $\left(U^{i j}\right)$ to the Christoffel symbols,

$$
\Gamma_{j k}^{i}:[0, \Lambda] \times B_{\vartheta}(0) \rightarrow F_{1}, \quad\left(\lambda,\left(U^{i \jmath}\right)\right) \rightarrow \Gamma_{j k}^{i},
$$

are of class $C^{\omega}$ for all $i, j, k=0,1,2,3$. Furthermore, there exists a constant $C$ such that for all $\left(U^{i j}\right) \in B_{\vartheta}(0)$ it holds that

$$
\begin{array}{ll}
\left\|\Gamma_{00}^{i}-U^{00}{ }_{, i}\right\|_{F_{1}} \leq C \lambda & \text { for } i \neq 0, \\
\left\|\Gamma_{j k}^{i}\right\|_{F_{1}} \leq C \lambda & \text { otherwise. }
\end{array}
$$



Proof. With the power series' of $\sqrt{|d|}-1$, and $\left(\bar{g}_{\imath \jmath}\right)-\left(\bar{g}_{i j}\right)$, it can be seen that the
functions

$$
\begin{aligned}
& {\left[\frac{1}{\lambda} \partial_{x^{2}} \sqrt{|d|}\right]:(-\Lambda, \Lambda) \times B_{\vartheta}(0) \rightarrow F_{1},} \\
& {\left[\frac{1}{\lambda} \partial_{x^{k}}\left(\bar{g}_{\imath \jmath}\right)\right]:(-\Lambda, \Lambda) \times B_{\vartheta}(0) \rightarrow F_{1}}
\end{aligned}
$$

are of class $C^{\omega}$. Furthermore, with (3.6) it follows that

$$
\begin{aligned}
& {\left[\frac{1}{\lambda} \partial_{x^{\imath}} \sqrt{|d|}\right]\left(0,\left(U^{\imath \jmath}\right)\right)=-2 U_{, i}^{00},} \\
& {\left[\frac{1}{\lambda} \partial_{x^{k}}\left(\bar{g}_{\imath j}\right)\right]\left(0,\left(U^{\imath \jmath}\right)\right)=-4\left(\delta_{i}^{0} \delta_{j}^{0}\right) U_{, k}^{00} .}
\end{aligned}
$$

Calculation of the Christoffel symbols yields

$$
\begin{aligned}
\Gamma_{\jmath k}^{\imath} & =\frac{1}{2} h^{i a}\left(h_{\jmath a, k}+h_{k a, j}-h_{\jmath k, a}\right), \\
h^{\imath \jmath} & =\frac{1}{\sqrt{|d|}} \bar{g}^{i j}, \\
h_{i j} & =\frac{1}{\lambda} \sqrt{|d|} \bar{g}_{i j} .
\end{aligned}
$$

Thus, the analyticity follows with the chain rule, the product rule, Proposition 3.10, and the continuity of

$$
\begin{aligned}
& \partial_{x^{i}}: F_{2} \rightarrow F_{1}, \\
& F_{2} \cdot F_{2} \rightarrow F_{2}, \\
& F_{1} \cdot F_{2} \rightarrow F_{1} .
\end{aligned}
$$

The equations $\left[h_{\imath \jmath, k}\right]_{\lambda=0}=-2 U^{00}{ }_{, k} \delta_{j}^{0} \delta_{i}^{0}$, and

$$
\left[h^{\imath j}\right]_{\lambda=0}=\underset{0}{\left.\left[\bar{g}^{\imath \jmath}\right)\right]_{\lambda=0}}=\operatorname{diag}(0,1,1,1)
$$

lead to

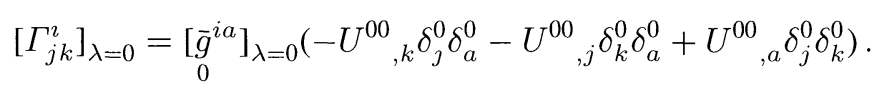

Thus, estimate (6.1) follows by Taylors theorem 3.7.

In order to prove Proposition 6.1, we choose $\Lambda$ such that the results of Sect. 3.3.2 can be used. Since the function space $C_{0}^{\infty}\left(\mathbb{R}^{3}\right)$ is dense in $F_{2}$, for all $\left(U^{\imath \jmath}\right)$ there exists a sequence $\left(\left(U^{\imath \jmath}\right)\right)_{n \in \mathbb{N}}$ of infinitely differentiable functions with compact support such that the estimates

$$
\left\|\left(\stackrel{n}{U^{\imath \jmath}}\right)-\left(U^{i j}\right)\right\|_{F_{2}^{*}} \leq \frac{1}{n}
$$

are fulfilled.

Using Proposition 3.10 and the results of Sect. 3.3.1, some straightforward considerations show that the maps

$$
\begin{aligned}
{\left[\frac{1}{\lambda^{2}}\left(G^{\imath \jmath}\right)\right]:[0, \Lambda] \times B_{\vartheta}(0) \rightarrow F_{0}^{*}, } & \left(\lambda,\left(U^{i j}\right)\right) & \rightarrow \frac{1}{\lambda^{2}}\left(G^{\imath \jmath}\right), \\
{\left[\left(F^{i j}\right)-\left(\Delta U^{\imath \jmath}\right)\right]:[0, \Lambda] \times B_{\vartheta}(0) \rightarrow F_{0}^{*}, } & \left(\lambda,\left(U^{\imath \jmath}\right)\right) & \rightarrow\left(F^{i j}\right)-\left(\Delta U^{i j}\right)
\end{aligned}
$$


are of class $C^{\omega}$. Here, Einstein's tensor $\left(G^{i j}\right)$, and $\left(F^{i j}\right)-\left(\Delta U^{i j}\right)$ are defined in (2.4), (2.3), respectively. It should be noted that $\frac{1}{\lambda^{2}}\left(G^{\imath \jmath}\right)$ is regular at $\lambda=0$. Thus, Taylors theorem 3.7 leads to the existence of a constant $M_{1}$ such that for all $0 \leq \lambda \leq \Lambda$ and all $\left(U^{\imath \jmath}\right)$ it holds that

$$
\begin{aligned}
& \left\|\left(F^{\imath \jmath}\right)-\left(\Delta U^{\imath j}\right)-\left(\stackrel{n}{F^{\imath j}}\right)+\left(\Delta \stackrel{n}{U}^{\imath \jmath}\right)\right\|_{F_{0}^{*}} \leq \frac{M_{1}}{n}, \\
& \left\|\frac{1}{\lambda^{2}}\left(G^{\imath \jmath}\right)-\frac{1}{\lambda^{2}}\left(\stackrel{n}{G^{2 \jmath}}\right)\right\|_{F_{0}^{*}} \leq \frac{M_{1}}{n} .
\end{aligned}
$$

Since a solution of the reduced field equations fulfills (4.4), it follows that

$$
U^{i j}(x)=\frac{-1}{4 \pi} \int_{\mathbb{R}^{3}} \frac{4 \pi G\left|d\left(x^{\prime}\right)\right| T^{i j}\left(x^{\prime}\right)-\stackrel{n}{F}^{\imath j}\left(x^{\prime}\right)+\Delta U^{\imath \jmath}\left(x^{\prime}\right)}{\left|x-x^{\prime}\right|} d^{3} x^{\prime}+\stackrel{n}{f^{i j}}(x)
$$

where

$$
\stackrel{n}{f}^{i j}(x)=\frac{-1}{4 \pi} \int_{\mathbb{R}^{3}} \frac{\stackrel{n}{F}^{i j}-\Delta \stackrel{n}{U}^{\imath j}-F^{i j}+\Delta U^{\imath j}}{\left|x-x^{\prime}\right|} d^{3} x^{\prime}
$$

With (3.1) and (6.2), it follows the existence of a constant $M_{2}$ such that

$$
\left\|\left(f^{i j}\right)\right\|_{F_{2}^{*}} \leq \frac{M_{2}}{n}
$$

This estimate and the continuity of $\partial_{x^{2}}: F_{2} \rightarrow F_{1}$ yield the existence of a constant $c$ such that for all $j=0,1,2,3$,

$$
\left\|f^{i j}{ }_{, \imath}\right\|_{F_{1}} \leq \frac{c}{n}
$$

The equation $\partial_{x^{k}}\left|x-x^{\prime}\right|=-\partial_{x^{\prime} k}\left|x-x^{\prime}\right|$ leads to

$$
\begin{aligned}
U^{i j}{ }_{, k}(x)= & \frac{1}{4 \pi} \int_{\mathbb{R}^{3}}\left(4 \pi G\left|d\left(x^{\prime}\right)\right| T^{i j}\left(x^{\prime}\right)-\stackrel{n}{F}^{\imath \jmath}\left(x^{\prime}\right)+\Delta \stackrel{n}{U}^{i j}\left(x^{\prime}\right)\right) \partial_{x^{\prime} k} \frac{1}{\left|x-x^{\prime}\right|} d^{3} x^{\prime} \\
& +f^{n}{ }_{, k}^{i j} .
\end{aligned}
$$

Since $|d|, T^{i j}$, and $\stackrel{n}{F}^{\imath \jmath}-\Delta \stackrel{n}{U^{\imath \jmath}}$ are continuously differentiable, we can apply Gauß' theorem. Because $\stackrel{n}{F^{i j}}-\Delta \stackrel{n}{U^{\imath \jmath}}$ has compact support, it holds that

$$
\begin{aligned}
U^{\imath \jmath}{ }_{, k}(x)= & \frac{-1}{4 \pi} \int_{\mathbb{R}^{3}} \frac{\partial_{x^{\prime} k}\left(4 \pi G\left|d\left(x^{\prime}\right)\right| T^{i j}\left(x^{\prime}\right)-\stackrel{n}{F}^{\imath \jmath}\left(x^{\prime}\right)+\Delta U^{\imath \jmath}\left(x^{\prime}\right)\right)}{\left|x-x^{\prime}\right|} d^{3} x^{\prime}+\stackrel{n}{f}_{, k}{ }_{, k} \\
& +\frac{1}{4 \pi} \int_{\partial T} \frac{4 \pi G\left|d\left(x^{\prime}\right)\right| T^{i j}\left(x^{\prime}\right) n_{k}\left(x^{\prime}\right)}{\left|x-x^{\prime}\right|} d \sigma\left(x^{\prime}\right) .
\end{aligned}
$$


Here, $d \sigma\left(x^{\prime}\right)$ denotes the infinitesimal surface element of $\partial T$. With $U_{{ }_{0}}^{i j}=T_{{ }_{0}}^{i j}=$ $\stackrel{n}{F}_{, 0}^{\imath \jmath}=\Delta \stackrel{n}{U}^{\imath \jmath}{ }_{, 0}=0$ and $\sum_{\imath=1}^{3} n_{\imath}\left(x^{\prime}\right) T^{i j}\left(x^{\prime}\right)=0$ we get

$$
\begin{aligned}
U^{\imath \jmath}{ }_{, i} & =U^{0 j}{ }_{, 0}+\sum_{i=1}^{3} U_{, i}^{i j} \\
& =\frac{-1}{4 \pi} \int_{\mathbb{R}^{3}} \frac{\left(4 \pi G\left|d\left(x^{\prime}\right)\right| T^{\imath \jmath}\left(x^{\prime}\right)-\stackrel{n}{F}^{i j}\left(x^{\prime}\right)+\Delta U^{i j}\left(x^{\prime}\right)\right)_{, i}}{\left|x-x^{\prime}\right|} d^{3} x^{\prime}+\stackrel{f}{f}_{, i}^{i j} .
\end{aligned}
$$

With

$$
\stackrel{n}{L}^{\imath \jmath}:=\bar{g}_{0}^{a b} \stackrel{n}{U}_{, a b}^{\imath \jmath}+\bar{g}_{0}^{i j}{\stackrel{n}{U^{a b}}, a b}_{, a b}^{2 U^{a(i}} \underset{, a b}{\bar{g}^{\jmath) b}}
$$

it follows that $\stackrel{n}{L}_{, \imath}^{i j}=0$, since $\left(\stackrel{n}{U}^{\imath \jmath}\right)$ is infinitely differentiable. This leads to

$$
U_{, i}^{\imath \jmath}=\frac{-1}{4 \pi} \int_{\mathbb{R}^{3}} \frac{\left(4 \pi G\left|d\left(x^{\prime}\right)\right| T^{i j}\left(x^{\prime}\right)-\stackrel{n}{F}^{\imath \jmath}\left(x^{\prime}\right)+\Delta \stackrel{n}{U}^{\imath \jmath}\left(x^{\prime}\right)-\stackrel{n}{L}^{i j}\left(x^{\prime}\right)\right)_{, \imath}}{\left|x-x^{\prime}\right|} d^{3} x^{\prime}+\stackrel{n}{f}_{f_{, \imath}} .
$$

Let $\left(\stackrel{n}{G^{i j}}\right)$ denote Einstein's tensor, defined by the tensor density $\left(\stackrel{n}{U^{\imath j}}\right)$. Then,

$$
\frac{|\stackrel{n}{d}|}{2 \lambda^{2}} \stackrel{n}{G} i j=\stackrel{n}{F} i j-\Delta \stackrel{n}{U^{i \jmath}}+\stackrel{n}{L^{\imath \jmath}}
$$

Thus,

$$
\begin{aligned}
U_{, i}^{\imath \jmath}(x)= & \frac{-1}{4 \pi} \int_{\mathbb{R}^{3}} \frac{4 \pi G\left|d\left(x^{\prime}\right)\right|{ }_{, \imath} T^{\imath \jmath}\left(x^{\prime}\right)-\frac{1}{2 \lambda^{2}}\left|{ }^{n} d\left(x^{\prime}\right)\right|{ }_{, \imath}{ }^{n}{ }^{i j}\left(x^{\prime}\right)}{\left|x-x^{\prime}\right|} d^{3} x^{\prime} \\
& +\frac{-1}{4 \pi} \int_{\mathbb{R}^{3}} \frac{4 \pi G\left|d\left(x^{\prime}\right)\right| T^{i j}{ }_{, i}\left(x^{\prime}\right)-\frac{1}{2 \lambda^{2}}\left|d\left(x^{\prime}\right)\right|{ }_{G^{\imath \jmath}}{ }_{, \imath}\left(x^{\prime}\right)}{\left|x-x^{\prime}\right|} d^{3} x^{\prime}+{ }_{f^{i j}}{ }_{, i}
\end{aligned}
$$

Since $\left(U^{\imath \jmath}\right)$ is a solution of the reduced field equations, and $U^{i j}{ }_{, 0}=0$, it holds that

$$
\begin{aligned}
4 \pi G T^{\imath \jmath}-\frac{1}{2 \lambda^{2}} G^{i j} & =\frac{1}{|d|}\left(\Delta U^{i j}-L^{i \jmath}\right)=\frac{1}{|d|}\left(\bar{g}_{0}^{a b} U_{, a b}^{i j}-L^{i j}\right) \\
& =-\frac{1}{|d|} H^{\imath \jmath},
\end{aligned}
$$

where

$$
H^{\imath \jmath}:=\bar{g}_{0}^{\imath \jmath} U_{a b}^{a b}-2 U^{a(i}{ }_{a b} \bar{g}_{0}^{j) b} .
$$

Let $\nabla_{\imath}$ denote the covariant derivative, and $\Gamma_{j k}^{i}$ the corresponding Christoffel symbols defined by the tensor density $\left(U^{i j}\right)$. Furthermore, $\stackrel{n}{\nabla}_{i}$, and $\stackrel{n}{\Gamma_{j k}^{i}}$ are defined by $\left(\stackrel{n}{U^{i j}}\right)$. 
Since $\nabla_{\imath} T^{i j}=0$ and $\stackrel{n}{\nabla} \stackrel{n}{G}^{i j}=0$, there follow

$$
4 \pi G|d|_{, \imath} T^{\imath \jmath}-\frac{1}{2 \lambda^{2}}|\stackrel{n}{d}|_{, \imath} \stackrel{n}{G}^{2 j}=-|d|_{, \imath} \frac{1}{|d|} H^{\imath \jmath}+|d|_{, i} \frac{1}{2 \lambda^{2}} G^{\imath \jmath}-\frac{1}{2 \lambda^{2}}|\stackrel{n}{d}|_{, \imath} \stackrel{n}{G}^{i j}
$$

and

$$
\begin{aligned}
& 4 \pi G|d| T_{, \imath}^{\imath \jmath}-\frac{1}{2 \lambda^{2}}|\stackrel{n}{d}| \stackrel{n}{G}_{, \imath}^{\imath \jmath} \\
& =4 \pi G|d| \nabla_{i} T^{\imath \jmath}-\frac{1}{2 \lambda^{2}} \stackrel{n}{\mid d} \mid \nabla_{\imath} \stackrel{n}{G}^{i j}-\Gamma_{\imath l}^{\imath}\left(4 \pi G|d| T^{l \jmath}-\frac{1}{2 \lambda^{2}}|\stackrel{n}{d}| \stackrel{n}{G^{l j}}\right) \\
& -\Gamma_{\imath l}^{j}\left(4 \pi G|d| T^{i l}-\frac{1}{2 \lambda^{2}}|\stackrel{n}{d}| \stackrel{n}{G}^{2 l}\right) \\
& =\frac{|\stackrel{n}{d}|}{2 \lambda^{2}}\left(\stackrel{n}{\nabla}_{\imath}-\nabla_{i}\right) \stackrel{n}{G^{\imath \jmath}}+\Gamma_{\imath l}^{\imath} H^{l j}+\Gamma_{\imath l}^{j} H^{i l} \\
& -\frac{1}{2 \lambda^{2}} \Gamma_{i l}^{2}\left(|d| G^{l j}-|\stackrel{n}{d}| \stackrel{n}{G^{l \jmath}}\right)-\frac{1}{2 \lambda^{2}} \Gamma_{\imath l}^{\jmath}\left(|d| G^{i l}-|\stackrel{n}{d}| \stackrel{n}{G^{2 l}}\right) \\
& =\frac{|\stackrel{n}{d}|}{2 \lambda^{2}}\left(\stackrel{n}{\Gamma}_{\imath l}^{i}-\Gamma_{i l}^{i}\right) \stackrel{n}{G}{ }^{l j}+\frac{|\stackrel{n}{d}|}{2 \lambda^{2}}\left(\stackrel{n}{\Gamma}_{i l}^{j}-\Gamma_{i l}^{j}\right) \stackrel{n}{G}{ }^{i l}+\Gamma_{\imath l}^{i} H^{l j}+\Gamma_{\imath l}^{\jmath} H^{i l} \\
& -\frac{1}{2 \lambda^{2}} \Gamma_{\imath l}^{i}\left(|d| G^{l \jmath}-|\stackrel{n}{d}| \stackrel{n}{G}{ }^{l j}\right)-\frac{1}{2 \lambda^{2}} \Gamma_{i l}^{\jmath}\left(|d| G^{i l}-|\stackrel{n}{d}| \stackrel{n}{G^{2 l}}\right) .
\end{aligned}
$$

Inserting (6.7) and (6.8) into (6.5) yields

$$
\begin{aligned}
U_{, i}^{i \jmath}(x)= & \frac{1}{4 \pi} \int_{\mathbb{R}^{3}} \frac{\left|d\left(x^{\prime}\right)\right|_{, i} \frac{1}{\left|d\left(x^{\prime}\right)\right|} H^{\imath \jmath}\left(x^{\prime}\right)}{\left|x-x^{\prime}\right|} d^{3} x^{\prime} \\
& +\frac{-1}{4 \pi} \int_{\mathbb{R}^{3}} \frac{\Gamma_{\imath l}^{\imath}\left(x^{\prime}\right) H^{l \jmath}\left(x^{\prime}\right)+\Gamma_{\imath l}^{j}\left(x^{\prime}\right) H^{i l}\left(x^{\prime}\right)}{\left|x-x^{\prime}\right|} d^{3} x^{\prime}+{\stackrel{n}{f^{i \jmath}}}_{, \imath}+\stackrel{n}{h}^{\jmath}(x),
\end{aligned}
$$

where

$$
\begin{aligned}
& h^{\jmath}(x) \\
& =\frac{-1}{4 \pi} \int_{\mathbb{R}^{3}} \frac{\frac{1}{2 \lambda^{2}}\left(\left|d\left(x^{\prime}\right)\right|_{, 2} G^{\imath j}\left(x^{\prime}\right)-\left|\stackrel{n}{d}\left(x^{\prime}\right)\right|_{, \imath} \stackrel{n}{G}^{\imath j}\left(x^{\prime}\right)\right)}{\left|x-x^{\prime}\right|} d f^{3} x^{\prime}
\end{aligned}
$$

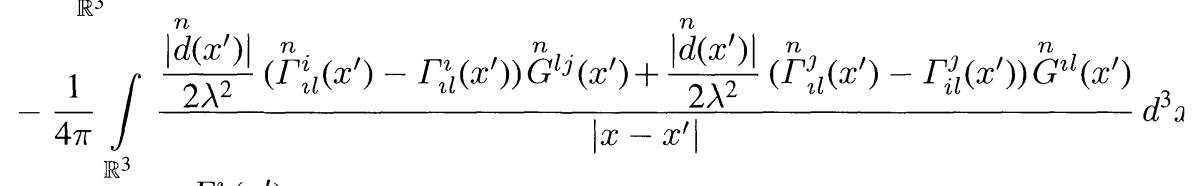

$$
\begin{aligned}
& -\frac{1}{4 \pi} \int_{\mathbb{R}^{3}} \frac{-\frac{\Gamma_{\imath l}^{\imath}\left(x^{\prime}\right)}{2 \lambda^{2}}\left(\left|d\left(x^{\prime}\right)\right| G^{l j}\left(x^{\prime}\right)-\left|{ }^{n}\left(x^{\prime}\right)\right|^{n} G^{l j}\left(x^{\prime}\right)\right)}{\left|x-x^{\prime}\right|} d^{3} x^{\prime} \\
& -\frac{1}{4 \pi} \int_{\mathbb{R}^{3}} \frac{-\frac{\Gamma_{i l}^{j}\left(x^{\prime}\right)}{2 \lambda^{2}}\left(\left|d\left(x^{\prime}\right)\right| G^{\imath l}\left(x^{\prime}\right)-\left.\left|{ }^{n} d\left(x^{\prime}\right)\right|\right|^{n}{ }^{2 l}\left(x^{\prime}\right)\right)}{\left|x-x^{\prime}\right|} d^{3} x^{\prime} .
\end{aligned}
$$


With the results of Sect. 3.3.1, the differentiability of $|d|-1, \Gamma_{\jmath k}^{i}$, and estimate (6.3) it follows the existence of a constant $c^{\prime}$ such that for all $j=0,1,2,3$ it holds that

$$
\left\|h^{n}(x)\right\|_{F_{2}} \leq \frac{c^{\prime}}{n}
$$

In order to prove the equation $U^{\imath j}{ }_{i}=0$, the following Banach spaces must be introduced:

Definition 6.3. For $p \geq 4$ and $0 \leq \delta<-2+3 \frac{p-1}{p}$ define

$$
F_{0}^{\prime}:=M_{0, \delta+1}^{p}\left(\mathbb{R}^{3}\right) \quad \text { and } \quad F_{1}^{\prime}:=M_{1, \delta}^{p}\left(\mathbb{R}^{3}\right) .
$$

With Proposition 3.3 and the definition of the weighted Sobolev spaces, we can easily convince ourselves that the embeddings

$$
\begin{aligned}
& F_{1} \rightarrow F_{0}^{\prime}, \quad F_{2} \rightarrow F_{1}^{\prime}, \\
& F_{0} \rightarrow F_{0}^{\prime}, \quad F_{1} \rightarrow F_{1}^{\prime},
\end{aligned}
$$

and the functions

$$
F_{1} \cdot F_{0}^{\prime} \rightarrow F_{0}, \quad \text { and } \quad \partial_{x^{2}}: F_{1}^{\prime} \rightarrow F_{0}^{\prime}
$$

are continuous.

We show that there are constants $\Lambda$ and $C$ such that for all $0 \leq \lambda \leq \Lambda$, all $n=1,2,3, \ldots$, and $j=0,1,2,3$ it holds that

$$
\left\|U^{i j}{ }_{, i}\right\|_{F_{1}^{\prime}} \leq \frac{C}{n} .
$$

Thus, a limit $n \rightarrow \infty$ yields $\left\|U^{i j}{ }_{, 2}\right\|_{F_{1}^{\prime}}=0$, which is equivalent to the desired result

$$
U_{, \imath}^{i j}=0 \text {. }
$$

Since $\left\|\left(U^{i j}\right)\right\|_{F_{2}^{*}} \leq \vartheta$ for all $0 \leq \lambda \leq \Lambda$, it holds that

$$
\alpha:=\sup \left\{\left\|U^{\imath \jmath}{ }_{, i}\right\|_{F_{1}^{\prime}} ; j=0,1,2,3\right\}<\infty .
$$

With the continuity of $\partial_{x^{2}}: F_{1}^{\prime} \rightarrow F_{0}^{\prime}$ and (6.6), the estimate

$$
\left\|H^{\imath \jmath}\right\|_{F_{0}^{\prime}} \leq C_{1} \alpha
$$

follows for an appropriate constant $C_{1}$. Furthermore, with the continuity of $\partial_{x^{2}}: F_{2} \rightarrow$ $F_{1}$ and (3.3) it follows the existence of a constant $C_{2}$ such that

$$
\left\||d|_{, \imath}\right\|_{F_{1}} \leq C_{2} \lambda \text {. }
$$

Finally, (3.1), Proposition 3.10, and the continuity of the multiplications $F_{2} \cdot F_{1} \rightarrow F_{1}$, $F_{1} \cdot F_{0}^{\prime} \rightarrow F_{0}$ lead to the existence of a constant $M$ such that for all $j=0,1,2,3$,

$$
\left\|\frac{1}{4 \pi} \int_{\mathbb{R}^{3}} \frac{\left|d\left(x^{\prime}\right)\right|_{, i} \frac{1}{\left|d\left(x^{\prime}\right)\right|} H^{\imath \jmath}\left(x^{\prime}\right)}{\left|x-x^{\prime}\right|} d^{3} x^{\prime}\right\|_{F_{2}} \leq M \lambda \alpha .
$$


With (6.1), the continuity of the multiplication $F_{1} \cdot F_{0}^{\prime} \rightarrow F_{0}$, and

$$
H^{00}=\bar{g}_{0}^{00} U_{, a b}^{a b}-2 U^{a(0}{ }_{a b}{ }_{0} \bar{g}^{0) b}=\lambda\left(U^{a 0}{ }_{a 0}-U^{a 1}{ }_{, a 1}-U^{a 2}{ }_{, a 2}-U^{a 3}{ }_{, a 3}\right),
$$

it follows the existence of a constant $C_{3}$ such that

$$
\left\|\Gamma_{i l}^{i} H^{l \jmath}+\Gamma_{i l}^{j} H^{\imath l}\right\|_{F_{0}} \leq C_{3} \lambda \alpha .
$$

This estimate and (3.1) yield the existence of a constant $M^{\prime}$ such that for all $j=0,1,2,3$,

$$
\left\|\frac{-1}{4 \pi} \int_{\mathbb{R}^{3}} \frac{\Gamma_{\imath l}^{\imath}\left(x^{\prime}\right) H^{l \jmath}\left(x^{\prime}\right)+\Gamma_{\imath l}^{\jmath}\left(x^{\prime}\right) H^{\imath l}\left(x^{\prime}\right)}{\left|x-x^{\prime}\right|} d^{3} x^{\prime}\right\|_{F_{2}} \leq M^{\prime} \lambda \alpha .
$$

Because $\|\cdot\|_{F_{1}^{\prime}} \leq\|\cdot\|_{F_{2}},\|\cdot\|_{F_{1}^{\prime}} \leq\|\cdot\|_{F_{1}}$, inserting (6.13), (6.14), (6.4), and (6.10) into Eq. (6.9) yields

$$
\begin{aligned}
\left\|U_{, \imath}^{\imath \jmath}(x)\right\|_{F_{1}^{\prime}} \leq & \left\|\frac{1}{4 \pi} \int_{\mathbb{R}^{3}} \frac{\left|d\left(x^{\prime}\right)\right|_{, \imath} \frac{1}{\left|d\left(x^{\prime}\right)\right|} H^{\imath \jmath}\left(x^{\prime}\right)}{\left|x-x^{\prime}\right|} d^{3} x^{\prime}\right\|_{F_{1}^{\prime}} \\
& +\left\|\frac{-1}{4 \pi} \int_{\mathbb{R}^{3}} \frac{\Gamma_{\imath l}^{i}\left(x^{\prime}\right) H^{l j}\left(x^{\prime}\right)+\Gamma_{i l}^{j}\left(x^{\prime}\right) H^{\imath l}\left(x^{\prime}\right)}{\left|x-x^{\prime}\right|} d^{3} x^{\prime}\right\|_{F_{1}^{\prime}} \\
& +\left\|f^{i \jmath}{ }_{, \imath}\right\|_{F_{1}^{\prime}}+\left\|h^{j}\right\|_{F_{1}^{\prime}} \\
\leq & \left(M+M^{\prime}\right) \lambda \alpha+\frac{c+c^{\prime}}{n} .
\end{aligned}
$$

such that

$$
\alpha:=\sup \left\{\left\|U_{, \imath}^{i j}\right\|_{F_{1}^{\prime}} ; j=0,1,2,3\right\} \leq\left(M+M^{\prime}\right) \lambda \alpha+\frac{c+c^{\prime}}{n} .
$$

$\Lambda<\left(M+M^{\prime}\right)^{-1}$, finally leads to (6.11), where $C=\frac{c+c^{\prime}}{1-\left(M+M^{\prime}\right) \Lambda}$.

\section{Result}

Propositions 4.2 and 6.1 yield the following

Theorem 7.1. Let $p_{\lambda}(\varrho)$ be a set of equations of state that fulfills the restrictions of Sect. 5.2. Assume that $T$ is a compact domain, and the density $\varrho_{0}: T \subset \mathbb{R}^{3} \rightarrow \mathbb{R}^{+}$is a solution of Euler's equations (5.3) in Newtonian fluid mechanics that represents a body rigidly rotating with angular velocity $\omega_{0}$ and consisting of ideal fluid with equation of state $p_{0}(\varrho)$. Furthermore,

- either the integral equation

$$
0=h_{0}(x)-F_{0}^{\prime}\left(A_{0}\left(\varrho_{0}(x)\right)\right) G \int_{T} h_{0}\left(x^{\prime}\right)\left(\frac{1}{\left|x-x^{\prime}\right|}-\frac{1}{\left|x^{\prime}\right|}\right) d^{3} x^{\prime}
$$


has the unique solution $h_{0}=0$ in

$$
C_{S}^{0}(T):=\left\{\begin{array}{l|l}
f \in C^{0}(T) & \begin{array}{l}
f \text { is symmetric relative to the } x^{3} \text {-axis } \\
f \text { is symmetric relative to the plane } x^{3}=0
\end{array}
\end{array}\right\},
$$

- or $\omega_{0}=0$, i.e. $\varrho_{0}$ represents a static, spherically symmetric star.

Then there are constants $\Lambda>0$ and $\Omega>0$ such that for all $0 \leq \lambda \leq \Lambda$ and all $|\delta \omega| \leq \Omega$ there exist a continuously differentiable density $\varrho: T_{\varrho} \rightarrow \mathbb{R}^{+}$and $a$ tensor density $\left(g^{\imath \jmath}\right)$ such that the stationary, axisymmetric Einstein equations (2.3) with causality constant $\lambda$ and energy-momentum tensor

$$
T^{i j}=\left(\varrho+\lambda p_{\lambda}(\varrho)\right) u^{2} v^{\imath} v^{j}+p_{\lambda}(\varrho) \frac{g^{\imath \jmath}}{\sqrt{\left|\operatorname{det}\left(g^{i \jmath}\right)\right|}}
$$

are fulfilled. Here, $T_{\varrho} \rightarrow \mathbb{R}^{3}$ is a bounded domain, and $u v^{i}$ is the velocity of the ideal fluid rotating with angular velocity $\omega_{0}+\delta \omega$ around the axis of symmetry. Additionally, it holds that $v=\partial_{t}+\left(\omega_{0}+\delta \omega\right) \partial_{\varphi}$, where $\partial_{t}$ is the timelike and $\partial_{\varphi}$ the spacelike Killing vector.

Furthermore, there exists a harmonic coordinate system $\left(x^{0}, x^{1}, x^{2}, x^{3}\right)$ such that

$$
v=\left(1,-\left(\omega_{0}+\delta \omega\right) x^{2},\left(\omega_{0}+\delta \omega\right) x^{1}, 0\right) .
$$

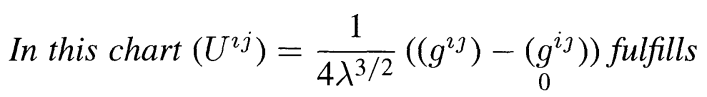

$$
\left(U^{\imath \jmath}\right) \in F_{2}^{*}, \quad U_{, \imath}^{i j}=0, \quad j=0,1,2,3,
$$

and the matter is symmetric relative to the $x^{3}$-axis and relative to the plane $x^{3}=0$.

Remark. I would like to mention, without proof, that the tensor density $\left(U^{i j}\right)$ is even analytic in the vacuum region. Furthermore, if $S$ is a compact domain in the star such that $F_{\lambda}, G_{\lambda} \in C^{l+1}(I)$, where

$$
I=\left\{y=-\frac{1}{2 \lambda} \ln \left(u_{\varrho, p}^{-2}(x)\right)+\frac{1}{2 \lambda} \ln \left(u_{\varrho, p}^{-2}(0)\right)+A_{\lambda}\left(\varrho_{0}(0)\right) \mid x \in S\right\},
$$

the density $\varrho$, the pressure $p$, and the tensor density $\left(U^{\imath \jmath}\right)$ are $l, l$, and $l+2$ times Hölder continuously differentiable in $S$, respectively.

Proof. In the case of a static star $\left(\omega_{0}=0\right), \varrho_{0}$ is spherically symmetric $[4,11]$, i.e. $\varrho_{0}$ depends only on $|x|$. Furthermore, it can be shown $[12,9]$ that in spherical coordinates

$$
\cos \vartheta, \quad \sin \vartheta \cos \varphi, \quad \text { and } \quad \sin \vartheta \sin \varphi
$$

is a basis of the kernel of $(\mathbb{1}-K): C^{0}(T) \rightarrow C^{0}(T)$, where $K$ is the compact operator

$$
\begin{aligned}
K: C_{S}^{0}(T) & \rightarrow C_{S}^{0}(T), \\
K: h(x) & \rightarrow[K(h)](x):=\frac{-G}{\Psi(x)} \int_{T}-\varrho_{0}^{\prime}\left(\left|x^{\prime}\right|\right) h\left(x^{\prime}\right)\left(\frac{1}{\left|x-x^{\prime}\right|}-\frac{1}{\left|x^{\prime}\right|}\right) d^{3} x^{\prime}
\end{aligned}
$$

with

$$
\Psi(x)=\frac{-4 \pi G}{|x|^{2}} \int_{0}^{|x|} \varrho_{0}(r) r^{2} d r, \quad \text { and } \quad \varrho_{0}^{\prime}(|x|)=\varrho_{0}^{\prime}(x)=\frac{x^{\imath}}{|x|} \partial_{x^{\imath}} \varrho_{0}(x)
$$


The equation $F_{0}^{\prime}\left(A_{0}\left(\varrho_{0}(x)\right)\right)=\left(A_{0}^{-1}\right)^{\prime}\left(A_{0}\left(\varrho_{0}(x)\right)\right)=\frac{1}{A_{0}^{\prime}\left(\varrho_{0}(x)\right)}$ and Euler's equation

$$
A_{0}^{\prime}\left(\varrho_{0}(x)\right) \partial_{x^{2}} \varrho_{0}(x)=\frac{-4 \pi G x^{i}}{|x|^{3}} \int_{0}^{|x|} \varrho_{0}(r) r^{2} d r
$$

yield

$$
F_{0}^{\prime}\left(A_{0}\left(\varrho_{0}(x)\right)\right)=\frac{\varrho_{0}^{\prime}(|x|)}{\Psi(x)}
$$

Thus, if there is any $h_{0} \in C_{S}^{0}(T)$ that fulfills Eq. (7.1), it follows that

$$
h_{0}(x)=\frac{\varrho_{0}^{\prime}(|x|)}{\Psi(x)} G \int_{T} h_{0}\left(x^{\prime}\right)\left(\frac{1}{\left|x-x^{\prime}\right|}-\frac{1}{\left|x^{\prime}\right|}\right) d^{3} x^{\prime}
$$

such that $\frac{h_{0}(x)}{\varrho_{0}^{\prime}(|x|)}$ lies in the kernel of $\mathbb{1}-K$. It should be noted that for any continuous function $h_{0}$ it holds that

$$
\frac{1}{\Psi(x)} G \int_{T} h_{0}\left(x^{\prime}\right)\left(\frac{1}{\left|x-x^{\prime}\right|}-\frac{1}{\left|x^{\prime}\right|}\right) d^{3} x^{\prime} \in C^{0}(T) .
$$

Thus, $\frac{h_{0}(x)}{\varrho_{0}^{\prime}(|x|)}$ can be extended continuously to the zeros of $\varrho_{0}^{\prime}(|x|)$. Consequently, in the case $\omega_{0}=0, h_{0}$ is a linear combination of

$$
\varrho_{0}^{\prime} \cos \vartheta, \quad \varrho_{0}^{\prime} \sin \vartheta \cos \varphi, \quad \text { and } \quad \varrho_{0}^{\prime} \sin \vartheta \sin \varphi
$$

which do not lie in $C_{S}^{0}(T)$.

Now we have convinced ourselves that in both cases the assumptions of Proposition 5.3 are fulfilled. Thus, for all $0 \leq \lambda \leq \Lambda$ and $\left|\omega-\omega_{0}\right| \leq \Omega$ there exists a tensor density $\left(U^{\imath \jmath}\right) \in F_{2}^{*}$ and a density $\varrho \in L^{p}\left(T \cup T_{\varepsilon}\right)$ such that for all $x \in T \cup T_{\varepsilon}$ there hold

$$
\begin{array}{r}
\varrho(x)=F_{\lambda}\left(-\frac{1}{2 \lambda} \ln \left(u^{-2}(x)\right)+\frac{1}{2 \lambda} \ln \left(u^{-2}(0)\right)+A_{\lambda}\left(\varrho_{0}(0)\right)\right) \\
p_{\lambda}(\varrho(x))=G_{\lambda}\left(-\frac{1}{2 \lambda} \ln \left(u^{-2}(x)\right)+\frac{1}{2 \lambda} \ln \left(u^{-2}(0)\right)+A_{\lambda}\left(\varrho_{0}(0)\right)\right) .
\end{array}
$$

Since $F_{\lambda}, G_{\lambda}$, and $\frac{1}{2 \lambda} \ln \left(u^{-2}(x)\right)$ are continuously differentiable, the density $\varrho$, the pressure $p$, and the energy-momentum tensor

$$
T^{i j}=\left(\varrho+\lambda p_{\lambda}(\varrho)\right) u^{-2} v^{\imath} v^{j}+p_{\lambda}(\varrho) \frac{g^{\imath \jmath}}{\sqrt{\left|\operatorname{det}\left(g^{i \jmath}\right)\right|}}
$$

are continuously differentiable. The surface of $T_{\varrho}$ is defined by

$$
f_{\lambda}\left(x_{0}\right)=-\frac{1}{2 \lambda} \ln \left(u^{-2}\left(x_{0}\right)\right)+\frac{1}{2 \lambda} \ln \left(u^{-2}(0)\right)+A_{\lambda}\left(\varrho_{0}(0)\right)=0
$$


and lies in $T \cup T_{\varepsilon}$. Because $f_{\lambda}$ is continuously differentiable, the surface $\partial T_{\varrho}$ is smooth, such that Gauß' theorem can be applied in $T_{\varrho}$. Furthermore, with (7.2), (7.3), $F_{\lambda}(0)=0$, and $G_{\lambda}(0)=0$ it follows that $T^{i j}\left(x_{0}\right)=0$ for all $x_{0} \in \partial T_{\varrho}$.

The fact that $\partial_{t}=(1,0,0,0), \partial_{\varphi}=\left(0,-x^{2}, x^{1}, 0\right)$, and $v^{2}$ are Killing vector was shown at the end of Sect. 4.2, such that Euler's equation (2.7) and the equations of motion $\nabla_{\imath} T^{\imath \jmath}=0$ are equivalent.

Thus, application of Proposition 6.1 yields that $\left(\lambda,\left(U^{i j}\right), T^{i j}\right)$ is a solution of Einstein's equations with causality constant $\lambda$.

Acknowledgements. I would like to thank H. Pfister, A.D. Rendall, and B.G. Schmidt for helpful suggestions and information about literature.

\section{References}

1. Adams, R.A.: Sobolev Spaces. New York, San Francisco, London: Academic Press 1975

2. Cantor, M.: Spaces of Functions with Asymptotic Conditions on $\mathbb{R}^{n}$. Indiana Univ. Math. J. 24(9), 897-902 (1975)

3. Cantor, M.: Elliptic Operators and the Decomposition of Tensorfields. Bull. Am. Math. Soc. 5, 235-262 (1981)

4. Carleman, T.: Über eine isoperimetrische Aufgabe und ihre physikalischen Anwendungen. Math. Z. 3, 1-7 (1919)

5. Chandrasekhar, S.: Ellipsoidal Figures of Equilibrium. New Haven, CT: Yale University Press, 1969

6. Deimling, K.: Nonlinear Functional Analaysis, Chap. 15, Berlin, Heidelberg, New York: Springer 1985

7. Ehlers, J.: Über den Newtonschen Grenzwert der Einsteinschen Gravitationstheorie. In: Nitsch, J., Pfarr, J., Stachow, E.-W., (eds.), Grundlagenprobleme der modernen Physik, 65-84, Mannheim, Wien, Zürich: Bibliographisches Institut 1981

8. Ehlers, J.: On Limit Relations Between, And Approximative Explanations Of, Physical Theories. In: Barcan Marcus, R., Dorn, G.J.W., Weingartner, P. (eds.) Logic, Methodology and Philosophy of Sience VII, 387-403, Amsterdam: North-Holland 1986

9. Heilig, U.: On Lichtenstein's Analysis of Rotating Newtonian Stars. To appear in Annales de L'Institut Henri Poincaré

10. Kreyszig, E.: Introductory Functional Analysis With Applications. Chap. 8, New York: John Wiley 1978

11. Lichtenstein, L.: Über eine isoperimetrische Aufgabe der mathematischen Physik. Math. Z. 3, 8-10 (1919)

12. Lichtenstein, L.: Gleichgewichtsfiguren rotierender Flüssigkeiten. Berlin: Springer 1933

13. Lindblom, L.: Fundamental Properties of Equilibrium Stellar Models. Ph.D. thesis, University of Maryland 1978

14. Lottermoser, M.: A Convergent Post-Newtonian Approximtion for the Constraint Equations in General Relativity. Annales de L'Institut Henri Poincaré 57, 279-317 (1992)

15. McOwen, R.C.: The Behaviour of the Laplacian on Weighted Sobolev Spaces. Comm. Pure Appl. Math. 32, 783-795 (1979)

16. Zeidler, E.: Vorlesung über nichtlineare Funktionsanalysis I. Chap. 4, Teubner Texte für Mathematik, 1975 
